This item was submitted to Loughborough's Research Repository by the author.

Items in Figshare are protected by copyright, with all rights reserved, unless otherwise indicated.

\title{
Holography, tomography and 3D microscopy as linear filtering operations
}

PLEASE CITE THE PUBLISHED VERSION

PUBLISHER

(C) Institute of Physics

LICENCE

CC BY-NC-ND 4.0

\section{REPOSITORY RECORD}

Coupland, Jeremy M., and J. Lobera. 2019. "Holography, Tomography and 3D Microscopy as Linear Filtering Operations". figshare. https://hdl.handle.net/2134/3361. 
This item was submitted to Loughborough's Institutional Repository by the author and is made available under the following Creative Commons Licence conditions.

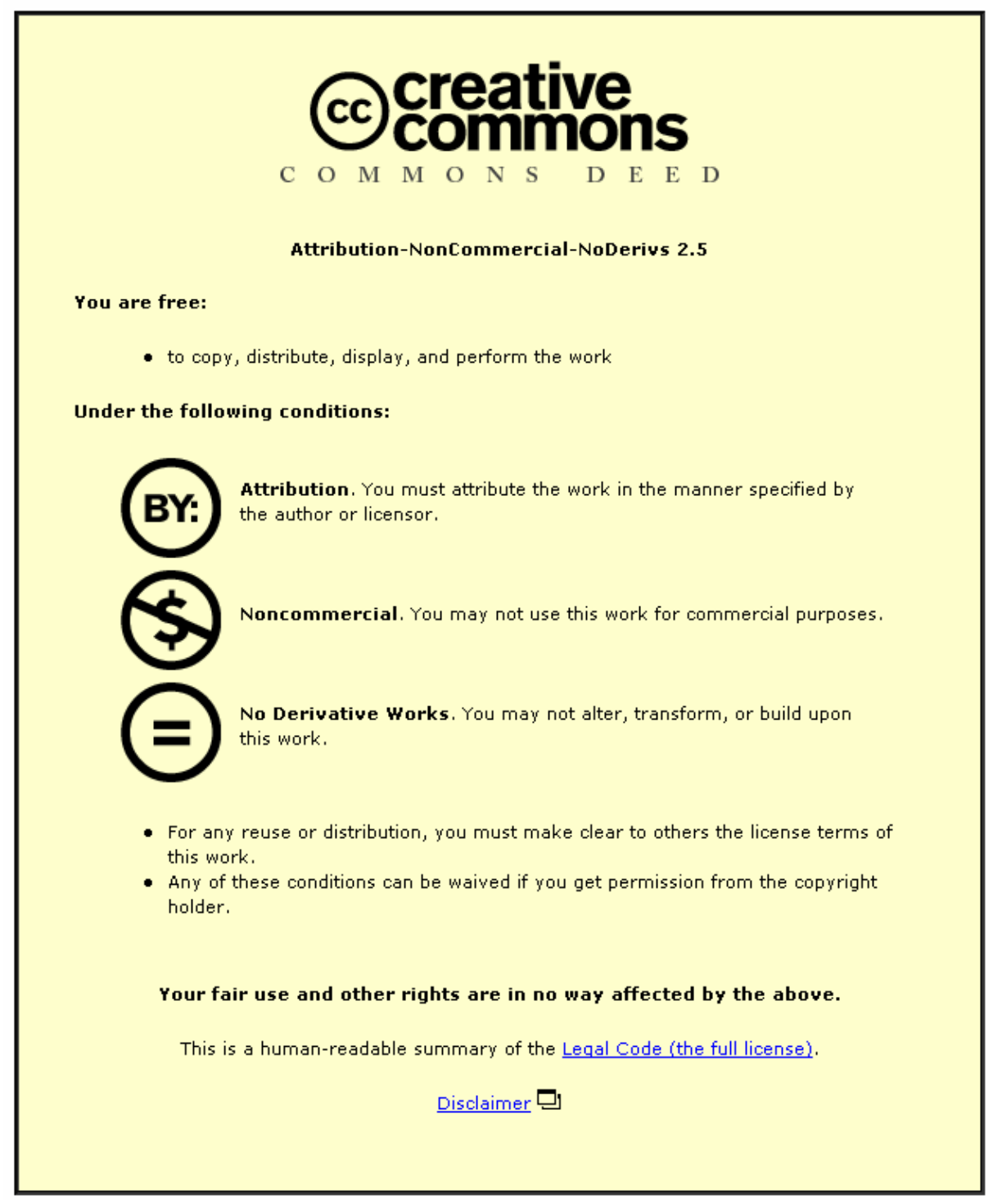

For the full text of this licence, please go to: http://creativecommons.org/licenses/by-nc-nd/2.5/ 


\title{
Holography, Tomography and 3D Microscopy as Linear Filtering Operations
}

\author{
J M Coupland and J Lobera \\ Wolfson School of Mechanical and Manufacturing Engineering \\ Loughborough University, Ashby Road, Loughborough, Leics. LE11 3TU \\ Email: J.M.Coupland@lboro.ac.uk
}

\begin{abstract}
In this paper we characterise 3D optical imaging techniques as 3D linear shift invariant filtering operations. From the Helmholtz equation that is the basis of scalar diffraction theory we show that the scattered field, or indeed a holographic reconstruction of this field, can be considered to be the result of a linear filtering operation applied to a source distribution. We note that if the scattering is weak, the source distribution is independent of the scattered field and a holographic reconstruction (or in fact any far-field optical imaging system) behaves as a 3D linear shift invariant filter applied to the refractive index contrast (which effectively defines the object). We go on to consider tomographic techniques that synthesise images from recordings of the scattered field using different illumination conditions. In our analysis we compare the $3 \mathrm{D}$ response of monochromatic optical tomography with the 3D imagery offered by confocal microscopy and scanning white light interferometry (using with quassi-monochromatic illumination) and explain the circumstances in which these approaches are equivalent. Finally, we consider the 3D response of polychromatic optical tomography and in particular the response of spectral optical coherence tomography and scanning white light interferometry.
\end{abstract}

Keywords: Holography, Digital Holography, Digital Holographic Microscopy, Optical Diffraction Tomography, Optical Coherence Tomography, Scanning White Light Interferometry, Confocal Microscopy, Impulse Response, Transfer function, Fourier Optics.

PACS: 42.30.-d,42.30.Kq,42.30.Lr,42.30.Wb,42.40.-I,42.62.Eh

\section{Introduction}

Three dimensional (3D) optical imaging has widespread application across a range of disciplines in engineering and medical diagnostics. In fluid mechanics pulsed holography has been used to record the position of particles in 3D space at one or more instants in time [1] and this is the basis of the flow mapping technique Holographic Particle Image Velocimetry (HPIV) [2]. Holographic Interferometry (HI) has been used to locate shock waves within gas turbines [3] and measure deformation of vibrating components in 3D [4]. In the electronics industry Scanning White Light Interferometry (SWLI) is commonly used to measure surface profile [5] and more recently film thickness [6]. In microbiology, the confocal microscope remains a popular method to provide sectioned images [7] while more recently Optical Diffraction Tomography (ODT) and Optical Coherence Tomography (OCT) have been used to great effect in microbial [8][9] and retinal imaging [10][11] respectively.

Although the techniques listed above might seem quite disparate both in configuration and application, they share a great deal in common. As optical techniques they all derive information purely from the 
way light is scattered from an object of interest and consequently have the advantage of being noncontacting. With the exception of the fluorescent imaging applications of confocal microscopy [12], elastically scattered light is collected and interferometric methods are used to record the phase and the amplitude of the scattered field. In an abstract sense, all of these imaging techniques measure the coherent response of an object to known electromagnetic stimuli in the visible spectrum. The difference between the techniques is essentially in the scanning methods used to provide the illuminating fields and those used to record the associated scattered fields.

Within the field of holography, the relationship between the physical characteristics of an object and the measured response was first considered by Wolf in 1969 [13] and shortly after by Dandliker and Weiss [14]. In these works, the plane wave components in monochromatic illumination and scattered fields are related to well-defined 3D spatial frequencies in the scattering potential (or equivalently in the refractive index contrast) that defines the object. They show that a linear relationship exists between these components for the case of weak scattering where multiple scattering can be neglected. Wolf's paper can be considered as the basis of optical tomography (more specifically ODT) and was subsequently demonstrated in practice by Fercher et al. [15]. If coherent recordings are made at more than one wavelength, then the technique is generally referred to as OCT and a similar relationship exists between plane wave components at various wavelengths and $3 \mathrm{D}$ spatial frequencies in the scattering potential [16].

In terms of linear systems theory, Wolf's work demonstrates that in certain circumstances, 3D image formation can be considered as a 3D linear shift invariant filtering process. As such, an optical system can be defined either by a point spread function in the space domain, or by a transfer function in the (spatial) frequency domain [17]. Characterisation of optical systems in these terms provides us with a means to compare their performance and we will make use of this approach extensively throughout this paper. The application of linear systems theory to optical systems is certainly not new, however, as it can be considered to be the basis of Fourier optics and has been applied extensively to 2D optical imaging [18]. The characterisation of 3D imaging techniques in this way differs fundamentally from the $2 \mathrm{D}$ case and should be approached with some caution. In practice, weak scattering is rarely observed in 3D objects and multiple scattering is usually significant. Despite this, linear inversion algorithms (that assume weak scattering) are almost exclusively used in commercial instrumentation and generally provide an initial estimate of the scattering potential in iterative, non-linear solvers [19].

In addition to the aforementioned work of Wolf and Fercher et al. in the fields of holography and optical tomography, several researchers have considered the 3D image formation in optical microscopes. Most notably, the 3D response of confocal microscopes has been studied extensively by Streibl [20] and Sheppard and Cogswell [21]. In this paper we analyse optical scattering and 3D imaging from first principles. We begin by considering the properties of the Helmholtz equation that is the basis of scalar diffraction and show that both the scattered field, and a holographic reconstruction of this field, can be considered to be the result of a 3D linear shift-invariant filtering applied to a source distribution. We note that if the scattering is weak, the source distribution is independent of the scattered field and a holographic reconstruction (or indeed that of any far-field optical imaging system) behaves as a 3D linear shift invariant filter applied to the refractive index contrast that effectively defines the object. Using this approach we characterise 3D optical imaging techniques and compare the attributes of each.

\section{Scalar Diffraction Theory}

We begin with a brief analysis of scalar diffraction theory. This offers a useful simplification of rigorous vector diffraction theory yet retains important features that allow us to compare 3D imaging techniques [22]. Accordingly the (complex) amplitude of a monochromatic electric field, E(r), propagating in a medium of (complex) refractive index, $\mathrm{n}(\mathbf{r})$, obeys the Helmholtz equation,

$$
\nabla^{2} \mathrm{E}(\mathbf{r})+\mathrm{k}_{0}^{2} \mathrm{n}^{2}(\mathbf{r}) \mathrm{E}(\mathbf{r})=0
$$

where $\mathrm{k}_{0}$ is the free space wave number defined here such that $\mathrm{k}_{0}=2 \pi / \lambda_{0}$, and $\lambda_{0}$ is the free space wavelength. In general, the electric field can be written as the superposition of, $\mathrm{E}^{\mathrm{r}}(\mathbf{r})$, the illuminating 
field (i.e. that which would be present if the object was absent) and, $E^{\mathrm{s}}(\mathbf{r})$, the scattered field. Although the object is usually defined by its scattering potential $\Phi(\mathbf{r})$, in this paper we prefer to define the object by the refractive index contrast $\Delta(\mathbf{r})=\Phi(\mathbf{r}) / 2 \mathrm{k}_{0}^{2}=\left(1-\mathrm{n}^{2}(\mathbf{r})\right) / 2$ since this is independent of wave number (in the absence of dispersion). Noting that $\left(\nabla^{2}+\mathrm{k}_{0}^{2}\right) \mathrm{E}^{\mathrm{r}}(\mathbf{r})=0$, the Helmholtz equation can then be written,

$$
\begin{aligned}
\left(\nabla^{2}+\mathrm{k}_{0}^{2}\right) \mathrm{E}^{\mathrm{s}}(\mathbf{r}) & =2 \mathrm{k}_{0}^{2} \Delta(\mathbf{r})\left(\mathrm{E}^{\mathrm{r}}(\mathbf{r})+\mathrm{E}^{\mathrm{s}}(\mathbf{r})\right) \\
& =\mathrm{U}(\mathbf{r})
\end{aligned}
$$

This equation is recognised as the free space scalar wave equation with source terms, $U(\mathbf{r})$, on the right-hand-side. In integral form the equation can be written as a convolution (denoted by $\otimes$ ), such that,

$$
\begin{aligned}
\mathrm{E}^{\mathrm{s}}(\mathbf{r}) & =\int_{-\infty}^{+\infty} \mathrm{G}\left(\mathbf{r}-\mathbf{r}^{\prime}\right) \mathrm{U}\left(\mathbf{r}^{\prime}\right) \mathrm{d}^{3} \mathrm{r}^{\prime} \\
& =\mathrm{G}(\mathbf{r}) \otimes \mathrm{U}(\mathbf{r})
\end{aligned}
$$

Here the function $G(\mathbf{r})$ is the well known, free-space Green's function that represents the field due to a unit point source of wave number $\mathrm{k}_{0}$. It is useful at this stage to examine the properties of this function. By definition the Green's function is a solution to the equation,

$$
\nabla^{2} \mathrm{G}(\mathbf{r})+\mathrm{k}_{0}^{2} \mathrm{G}(\mathbf{r})=-\delta(\mathbf{r})
$$

where $\delta(\mathbf{r})$ is the Dirac delta function. In the spatial frequency domain, which we will henceforth refer to as k-space, an expression for the Green's function can be found directly. Fourier transformation of equation 4 gives,

$$
\widetilde{\mathrm{G}}(\mathbf{k})=\frac{1}{|\mathbf{k}|^{2}-\mathrm{k}_{0}^{2}}
$$

where tilde $(\sim)$ denotes Fourier transformation and $\mathbf{k}$ denotes spatial frequency. Inverse Fourier transformation of this expression yields the familiar space domain definition of a source,

$$
\mathrm{G}(\mathbf{r})=\frac{\mathrm{e}^{\mathrm{j} \mathrm{k}_{0}|\mathbf{r}|}}{4 \pi|\mathbf{r}|}
$$

The 2D free-space Green's function is illustrated in figure 1a) and b). In the space domain (figure 1a) the Green's function has a singular point at the origin but appears wave-like elsewhere. In k-space it can be seen that the Green's function is dominated by its value on an infinitely thin, shell defined by $|\mathbf{k}|=\mathrm{k}_{0}$ which in $3 \mathrm{D}$ is a spherical shell that we will refer to as the Ewald sphere.
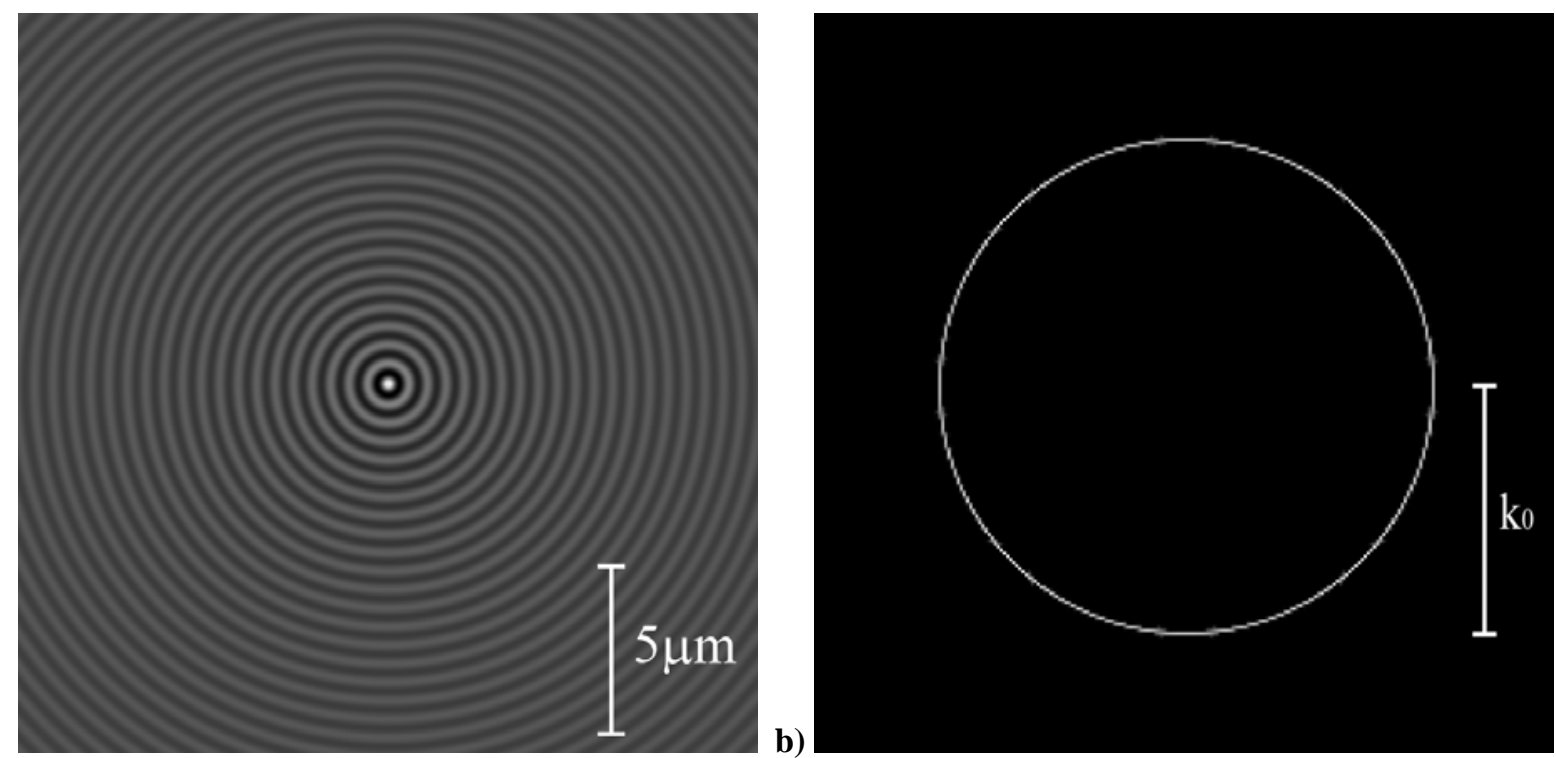

b) 
Figure 1 Free space Green’s function in a) space domain (real part) b) k-space (absolute value).

In the terms of linear systems theory, the scattered field, $E^{s}(\mathbf{r})$, that is defined by equation 3 , is the result of a linear shift invariant filtering operation applied to the source distribution, $\mathrm{U}(\mathbf{r})$. As such it is characterised either in $\mathrm{k}$-space by the transfer function, or in the space domain by the impulse response function. In this case, these functions are simply the free-space Green's function defined in equations 5 and 6 respectively.

\section{Holographic Recording and Reconstruction}

Holographic reconstruction has been considered by many authors in a variety of different recording geometries [23]. It is noted, however, that all holographic reconstructions, regardless of geometry, are based purely on measurements of the scattered field on a surface (CCD or holographic plate) that is placed at some distance from the object. As a direct consequence of this we shall show that a holographic reconstruction cannot be an accurate representation of the field within regions of inhomogeneity. More generally we will show that a holographic reconstruction is a filtered version of the scattered field within such regions.

Let us consider the scattering geometry shown in figure 2. Without loss of generality, we have assumed that the origin of our coordinate system is found within a relatively small region of inhomogeneity and consider the field at a relatively large boundary surface that encloses this region.

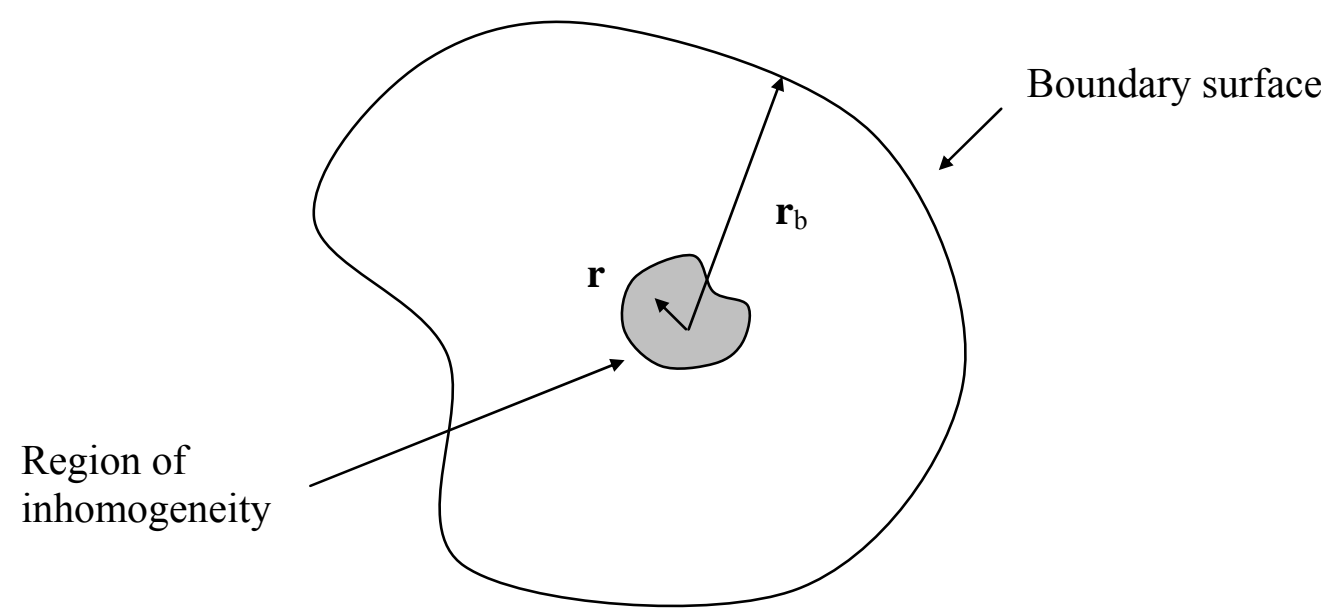

Figure 2 Scattering Geometry

We note that the Green's function that defines the field at a point on the boundary, $\mathrm{r}_{0}$, due to a unit source at position, $r$, can be simplified when $\left|\mathbf{r}_{\mathrm{b}}\right|>>|\mathbf{r}|$, whence we find,

$$
\mathrm{G}\left(\mathbf{r}-\mathbf{r}_{\mathrm{b}}\right)=\frac{\mathrm{e}^{\mathrm{k}_{0}\left|\mathbf{r}-\mathbf{r}_{\mathrm{b}}\right|}}{4 \pi\left|\mathbf{r}-\mathbf{r}_{\mathrm{b}}\right|} \approx \frac{\mathrm{e}^{\mathrm{j} \mathrm{k}_{0}\left|\mathbf{r}_{\mathrm{b}}\right|}}{4 \pi\left|\mathbf{r}_{\mathrm{b}}\right|} \mathrm{e}^{-\mathrm{j} \frac{\mathrm{k}_{0}}{\left|\mathbf{r}_{\mathrm{b}}\right|} \mathbf{r} \cdot \mathbf{r}_{\mathrm{b}}}
$$

In essence this is the Fraunhofer approximation [18] and making this assumption, from equation 3 the boundary field can be written,

$$
E^{\mathrm{s}}\left(\mathbf{r}_{\mathrm{b}}\right)=\frac{\mathrm{e}^{\mathrm{j} \mathrm{k}_{0}\left|\mathbf{r}_{\mathrm{b}}\right|}}{4 \pi\left|\mathbf{r}_{\mathrm{b}}\right|} \int_{-\infty}^{+\infty} U(\mathbf{r}) \mathrm{e}^{-\mathrm{j} \frac{\mathrm{k}_{0}}{\mid \mathbf{r}_{\mathrm{b}}} \mathbf{r} \cdot \mathbf{r}_{\mathrm{b}}} \mathrm{d}^{3} \mathrm{r}
$$

Equation 8 shows that the field at a distant boundary provides an estimate of the source spectrum, $\tilde{U}(\mathbf{k})$, and we may write, 


$$
\widetilde{\mathrm{U}}\left(\frac{\mathrm{k}_{0} \mathbf{r}_{\mathrm{b}}}{\left|\mathbf{r}_{\mathrm{b}}\right|}\right)=4 \pi\left|\mathbf{r}_{\mathrm{b}}\right| \mathrm{e}^{-\mathrm{j} \mathrm{k}_{0}\left|\mathbf{r}_{\mathrm{b}}\right|} \mathrm{E}^{\mathrm{s}}\left(\mathbf{r}_{\mathrm{b}}\right)
$$

It is noted however, that since the vector $\mathbf{r}_{\mathbf{b}} /\left|\mathbf{r}_{\mathbf{b}}\right|$, has unit magnitude, the boundary field provides only spectral values when $|\mathbf{k}|=\mathrm{k}_{0}$ (i.e. those on the surface of the Ewald sphere) and, in general, a holographic image can be thought of as a reconstruction based on this partial information. Accordingly, we can express the reconstructed field, $\mathrm{E}^{\mathrm{m}}(\mathbf{r})$, as the Fourier integral,

$$
\mathrm{E}^{\mathrm{m}}(\mathbf{r})=\int_{-\infty}^{+\infty} \frac{\delta\left(|\mathbf{k}|-\mathrm{k}_{0}\right)}{\mathrm{k}_{0}^{2}} \widetilde{\mathrm{U}}(\mathbf{k}) \mathrm{e}^{-\mathrm{jk} \cdot \mathbf{r}} \mathrm{d}^{3} \mathrm{k}
$$

where the factor $1 / k_{0}^{2}$ is used to normalize the measurement. Equivalently in k-space we can write,

$$
\widetilde{\mathrm{E}}^{\mathrm{m}}(\mathbf{k})=\frac{\delta\left(|\mathbf{k}|-\mathrm{k}_{0}\right)}{\mathrm{k}_{0}^{2}} \widetilde{\mathrm{U}}(\mathbf{k})
$$

With reference to our discussions of linear filtering in the previous section, we can summarize the whole process of holographic reconstruction as a linear shift invariant filtering operation applied to the source distribution. In this case an ideal optical system that records the field over a complete closed boundary has a transfer function, $\widetilde{\mathrm{G}}_{\text {ideal }}(\mathbf{k})$, that is given by,

$$
\widetilde{\mathrm{G}}_{\text {ideal }}(\mathbf{k})=\frac{\delta\left(|\mathbf{k}|-\mathrm{k}_{0}\right)}{\mathrm{k}_{0}^{2}}
$$

In the space domain the impulse response, $\mathrm{G}_{\text {ideal }}(\mathbf{r})$, is given by

$$
\begin{aligned}
\mathrm{G}_{\text {ideal }}(\mathbf{r}) & =\frac{1}{\mathrm{k}_{0}^{2}} \int_{-\infty}^{+\infty} \delta\left(|\mathbf{k}|-\mathrm{k}_{0}\right) \mathrm{e}^{\mathrm{j} \mathbf{k} \cdot \mathbf{r}} \mathrm{d}^{3} \mathrm{k} \\
& =\operatorname{sinc}\left(\mathrm{k}_{0}|\mathbf{r}|\right)
\end{aligned}
$$

Although this analysis presented above is based on the Fraunhofer approximation that was used to simplify the Green's function of equation 7, it is far more general since the field at infinity can be calculated from that measured on any other closed boundary. In fact, additional spectral information only becomes available when the boundary is sufficiently close to the source distribution for evanescent waves to make a significant contribution [22].

In practice measurements are typically made over a finite region of the Ewald sphere and we can modify our approach to include this constraint. For an axial system with finite Numerical Aperture (NA) the corresponding transfer function, $\widetilde{\mathrm{G}}_{\mathrm{NA}}(\mathbf{k})$, can be written,

$$
\widetilde{\mathrm{G}}_{\mathrm{NA}}(\mathbf{k})=\frac{1}{\mathrm{k}_{0}^{2}} \delta\left(|\mathbf{k}|-\mathrm{k}_{0}\right) \operatorname{step}\left(\mathbf{k} . \hat{\mathbf{o}}-\mathrm{k}_{0} \sqrt{1-\mathrm{NA}^{2}}\right)
$$

where $\hat{\mathbf{o}}$ is a unit vector in the direction of the viewing axis and step(x) is the Heaviside Step Function [24]. Because of the importance of this transfer function we now consider its form in some detail. A schematic diagram showing the non-zero part of the transfer function is shown in figure 3. 


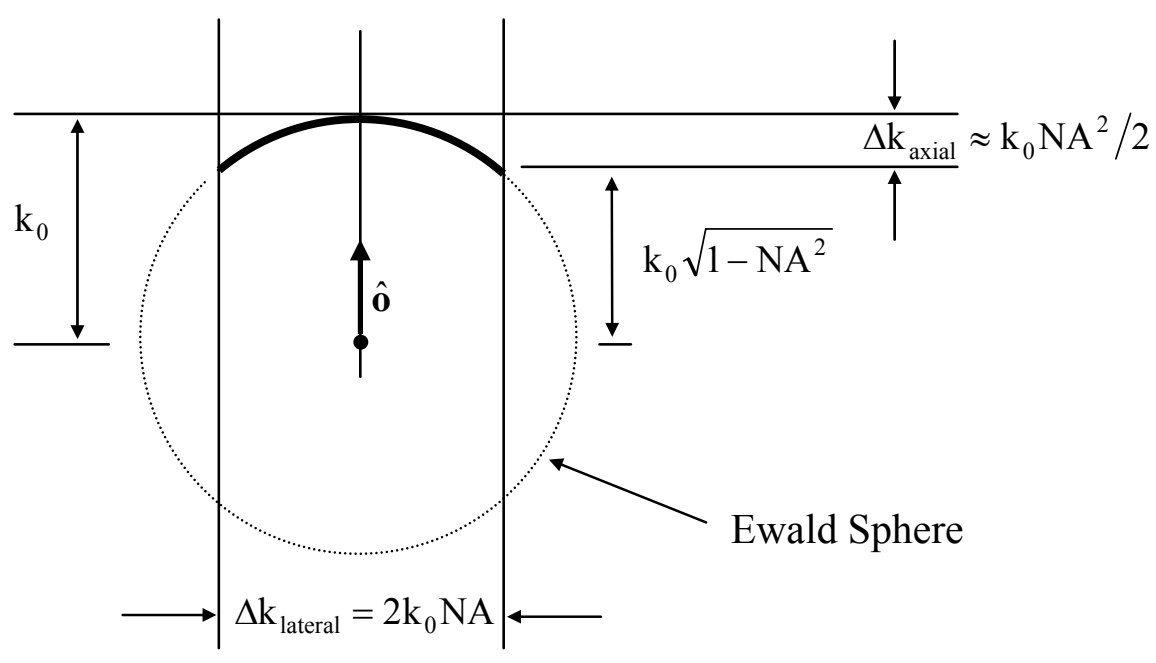

Figure 3 Transfer function with restricted NA

In the axial (viewing) direction it can be seen that the bandwidth of the transfer function, $\Delta \mathrm{k}_{\text {axial }}$ depends strongly on the NA and is given by,

$$
\begin{aligned}
\Delta \mathrm{k}_{\text {axial }} & =\mathrm{k}_{0}\left(1-\sqrt{1-\mathrm{NA}^{2}}\right) \\
& \approx \frac{\mathrm{k}_{0} \mathrm{NA}^{2}}{2}
\end{aligned}
$$

In contrast the lateral bandwidth, $\Delta \mathrm{k}_{\text {lateral }}$, is directly proportional to the NA of the optical system,

$$
\Delta \mathrm{k}_{\text {lateral }}=2 \mathrm{k}_{0} \mathrm{NA}
$$

By definition the impulse response is given by

$$
\mathrm{G}_{\mathrm{NA}}(\mathbf{r})=\frac{1}{\mathrm{k}_{0}^{2}} \int_{-\infty}^{+\infty} \delta\left(|\mathbf{k}|-\mathrm{k}_{0}\right) \operatorname{step}\left(\mathbf{k} . \hat{\mathbf{o}}-\mathrm{k}_{0} \sqrt{1-\mathrm{NA}^{2}}\right) \mathrm{e}^{\mathrm{j} \mathbf{k} \cdot \mathbf{r}} \mathrm{d}^{3} \mathrm{k}
$$

To the Author's knowledge this integral has no closed form solution, however, some insight into the impulse response can be gained by considering change in phase of the integrand with position, $\mathbf{r}$. According to Rayleigh's quarter wave rule [22], the value of the integral will not depart significantly from its value at the origin if the phase of the integrand is bounded by $\mathbf{r} . \Delta \mathbf{k}<\pi / 2$. This provides us with one measure of resolution, It is more usual however, to define resolution in terms of the Nyquist distance that corresponds to the shortest spatial period that can be faithfully reproduced by a system of a given bandwidth [8]. We note that for the the case of a uniformly sampled 1D distribution this corresponds to the Rayleigh resolution [22]. If $\mathbf{r}$ is less than the Nyquist distance we find that the phase of the integrand in equation 17 is bounded such that $\mathbf{r} . \Delta \mathbf{k}<2 \pi$. With reference to bandwidths defined in figure 3 the axial extent of the impulse response or resolution in the axial direction $\Delta r_{\text {axial }}$ is then estimated to be,

$$
\Delta \mathrm{r}_{\text {axial }}=\frac{2 \pi}{\Delta \mathrm{k}_{\text {axial }}} \approx \frac{2 \lambda}{\mathrm{NA}^{2}}
$$

Similarly the lateral extent of the impulse response function or the axial resolution $\Delta \mathrm{r}_{\text {lateral }}$ is estimated to be,

$$
\Delta \mathrm{r}_{\text {lateral }}=\frac{2 \pi}{\Delta \mathrm{k}_{\text {lateral }}}=\frac{\lambda}{2 \mathrm{NA}}
$$

Using numerical methods to solve equation 2 together with the filtering operation defined by the transfer function in equation 14 we are able to simulate scattered fields and holographic reconstructions. We illustrate the process of holographic reconstruction for the case of a single 
particle. The particle is assumed to be water $(\mathrm{n}=1.33)$ in ambient air and is $2 \mu \mathrm{m}$ in diameter. For the purposes of illustration the scattered field is calculated in 2D using an FEM solution to equation 2. The absolute value of the scattered field is shown in figure $4 \mathrm{a}$ ) for the case of plane wave illumination that propagates from the bottom of the figure.

a)
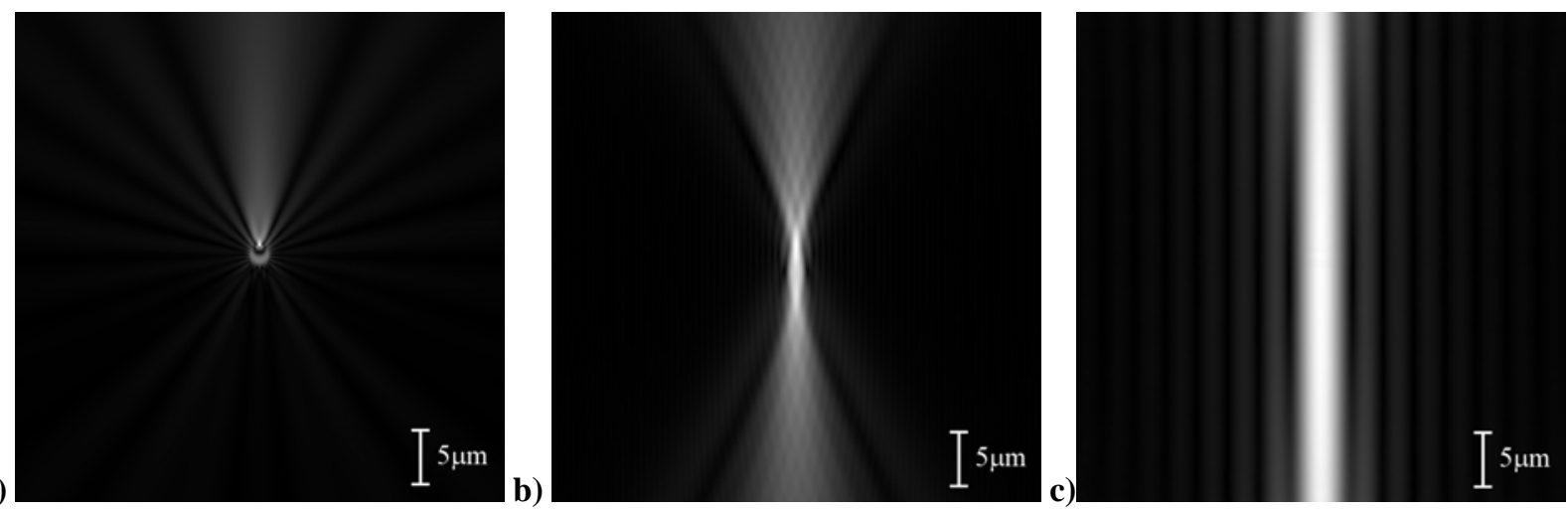

Figure 4 Two micron particle: a) calculated scattered field, b) reconstructed fields for NA=0.5 and c) for NA=0.1 (absolute value).

Figures $4 \mathrm{~b}$ ) and $4 \mathrm{c}$ ) illustrate the filtering introduced when the reconstruction optics have NA of $\mathrm{NA}=0.5$ and $\mathrm{NA}=0.1$ respectively. It is noted immediately, that in both cases the reconstruction process introduces a significant field "upstream" of the scattering particle (i.e. towards the source of illumination). It is also noted that detail is absent from the reconstruction and in the low NA=0.1 case of figure $4 \mathrm{c}$ ), the particle is not properly resolved and consequently the image closely resembles the elongated impulse response of the optical system.

Up to this point we have only considered the performance of an optical system that is used for observation. We have shown from first principles that such a system can be characterized as a linear filter with a transfer function that depends strongly on the NA of the recording system. It is important to note that the filtering operation is applied to the source distribution and not the refractive index contrast that we use to define the object. In general, the source distribution is a non-linear function of the contrast since (from equation 2) the former depends on both the illumination and the scattered fields. Measurements of the scattered field when an object is illuminated by one or more reference fields can be related linearly to the contrast if the scattering is weak. We now consider this case in more detail.

\section{Monochromatic Optical Tomography}

Linear optical tomography is based on the assumption that the illuminating field is not affected by the object itself. In this case the source distribution, $\mathrm{U}(\mathbf{r})$, can be written,

$$
\mathrm{U}(\mathbf{r})=2 \mathrm{k}_{0}^{2} \Delta(\mathbf{r})\left(\mathrm{E}^{\mathrm{r}}(\mathbf{r})+\mathrm{E}^{\mathrm{s}}(\mathbf{r})\right) \approx 2 \mathrm{k}_{0}^{2} \Delta(\mathbf{r}) \mathrm{E}^{\mathrm{r}}(\mathbf{r})
$$

This is the First Born Approximation [22] and is valid if the contrast is small or if the object consists of sparse point-like objects (as is generally the case in Holographic Particle Image Velocimetry). Making this approximation a holographic reconstruction takes the form,

$$
\mathrm{E}^{\mathrm{m}}(\mathbf{r})=2 \mathrm{k}_{0}^{2} \int_{-\infty}^{+\infty} \mathrm{G}_{\mathrm{NA}}\left(\mathbf{r}-\mathbf{r}^{\prime}\right) \Delta\left(\mathbf{r}^{\prime}\right) \mathrm{E}^{\mathrm{r}}\left(\mathbf{r}^{\prime}\right) \mathrm{d}^{3} \mathrm{r}^{\prime}
$$

In order to calculate the contrast function, the reconstruction is demodulated by multiplying by the conjugate of the illuminating wave such that,

$$
\mathrm{E}^{\mathrm{m}}(\mathbf{r}) \mathrm{E}^{\mathrm{r}}(\mathbf{r})^{*}=2 \mathrm{k}_{0}^{2} \int_{-\infty}^{+\infty} \mathrm{G}_{\mathrm{NA}}\left(\mathbf{r}-\mathbf{r}^{\prime}\right) \Delta\left(\mathbf{r}^{\prime}\right) \mathrm{E}^{\mathrm{r}}\left(\mathbf{r}^{\prime}\right) \mathrm{E}^{\mathrm{r}}(\mathbf{r})^{*} \mathrm{~d}^{3} \mathrm{r}^{\prime}
$$

If the illumination is a plane wave such that $\mathrm{E}^{\mathrm{r}}(\mathbf{r})=\mathrm{e}^{\mathrm{j} \mathbf{k}_{\mathrm{i}} \cdot \mathbf{r}}$, the reconstructed image $\mathrm{O}_{\text {Holo }}(\mathrm{r})$, can be written,

$$
\mathrm{O}_{\text {Holo }}(\mathbf{r})=\mathrm{E}^{\mathrm{m}}(\mathbf{r}) \mathrm{e}^{-\mathrm{j} \mathbf{k}_{\mathrm{i}} \cdot \mathbf{r}}=2 \mathrm{k}_{0}^{2} \int_{-\infty}^{+\infty} \mathrm{G}_{\mathrm{NA}}\left(\mathbf{r}-\mathbf{r}^{\prime}\right) \mathrm{e}^{\mathrm{j} \mathbf{k}_{\mathrm{i}} \cdot\left(\mathbf{r}-\mathbf{r}^{\prime}\right)} \Delta\left(\mathbf{r}^{\prime}\right) \mathrm{d}^{3} \mathbf{r}^{\prime}
$$


Thus subject to the Born Approximation the demodulated measurement of the scattered field can be considered as the result of a linear shift invariant filtering operation. It is noted that the impulse response is now applied to the refractive index contrast rather than to the source function as in previous sections and we will use the letter ' $H$ ' rather than ' $G$ ' to emphasise this difference. The impulse response of such a reconstruction which we will refer to as, $\mathrm{H}_{\mathrm{Holo}}(\mathbf{r})$, is given by,

$$
\mathrm{H}_{\mathrm{Holo}}(\mathbf{r})=2 \mathrm{k}_{0}^{2} \mathrm{G}_{\mathrm{NA}}(\mathbf{r}) \mathrm{e}^{\mathrm{j} \mathbf{k}_{\mathrm{i}} \cdot \mathbf{r}}
$$

In k-space the transfer function is,

$$
\widetilde{\mathrm{H}}_{\mathrm{Holo}}(\mathbf{k})=2 \mathrm{k}_{0}^{2} \widetilde{\mathrm{G}}_{\mathrm{NA}}\left(\mathbf{k}-\mathbf{k}_{\mathrm{i}}\right)
$$

In figures $5 \mathrm{a}$ ) and $5 \mathrm{~b}$ ) the transfer functions are illustrated in $2 \mathrm{D}$ for the case an objective with an $\mathrm{NA}=0.5$ for back-scatter and forward-scatter respectively.

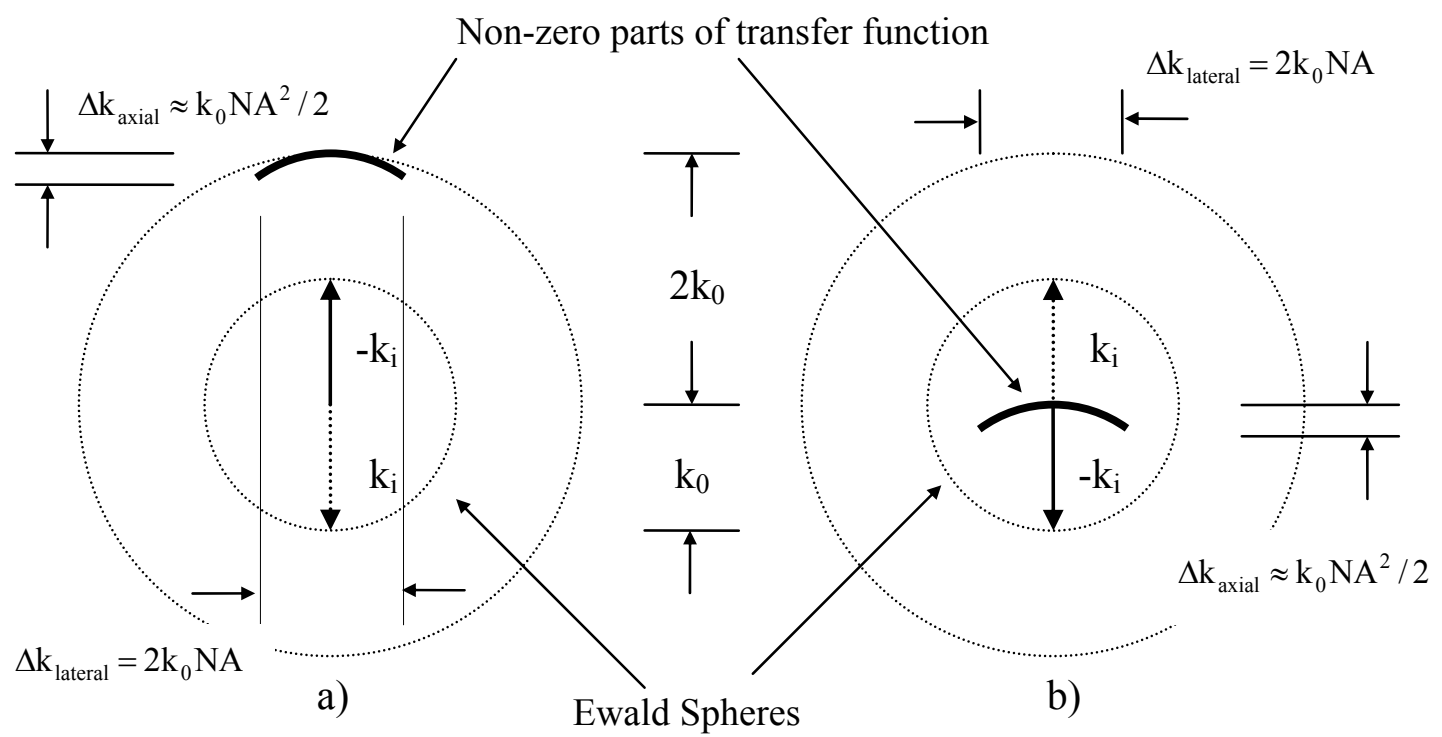

Figure 5 Transfer functions for a) back-scatter and b) forward-scatter geometry.

It can be seen that in the back-scatter geometry the system is responsive to high spatial frequencies and consequently large gradients caused for example by step changes in refractive index. In contrast, the forward-scatter geometry is only responsive to low spatial frequencies in the axial direction. The corresponding impulse responses have been calculated for the case of NA=0.5 and $\lambda=550 \mathrm{~nm}$ and the real values of these are shown in figures $6 a$ ) and $6 \mathrm{~b}$ ).
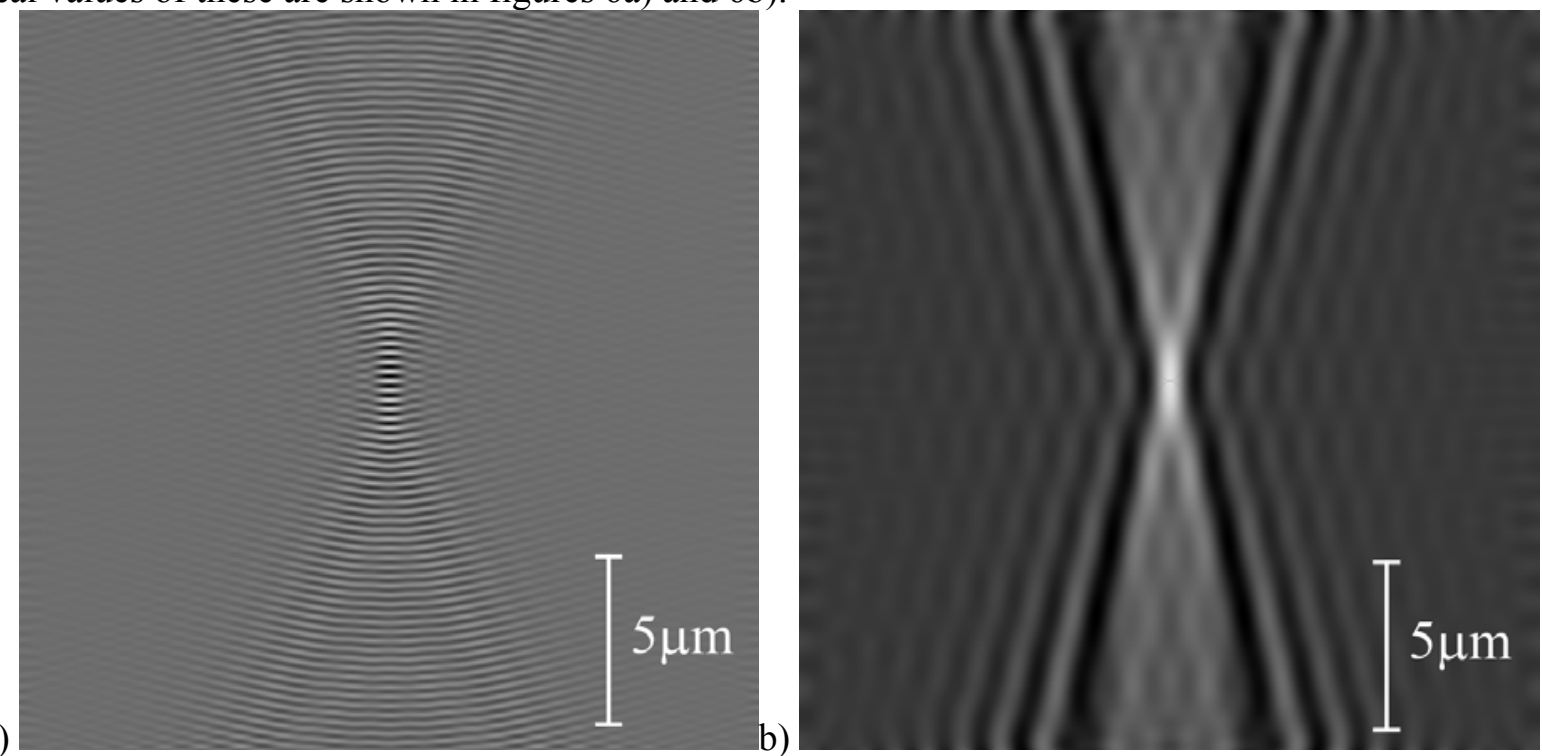

Figure 6 Impulse response functions for a) back-scatter and b) forward-scatter geometry (real part). 
It is noted that in each case the impulse response decays relatively slowly with radial distance. In fact the squared magnitude of the response falls off as the inverse square of axial distance (in both forward and back-scatter) and this means that the total energy flux is equal for any closed surface that bounds the region of inhomogeneity. It has been argued elsewhere [15] that because of this, a reconstruction that is made from a single holographic record contains no 3D information. If we consider for a moment reconstructions made of particulate holograms, however, it is clear that it is generally possible to identify individual particles and estimate their position in 3D space providing a finite region of space is seeded and the seeding is sufficiently sparse. It is noted that in HPIV, the relatively slow decay of the impulse response ultimately restricts the number of velocity measurements that can be made for a given flow geometry [25].

Synthesis of an impulse response function with a much more rapid axial decay can be achieved however, by combining the effect of many recordings with different illumination/observation geometries. This approach effectively distinguishes between holography and tomography. It is clear that there are many different ways to achieve this and we will compare the main strategies in the following sections.

\subsection{Lauer Microscopy}

First we consider the case of a single observation geometry and a set of plane wave illuminations. This technique was that originally explored by Wolf [13] and has subsequently been demonstrated to great effect by Lauer [8]. We will refer to the technique as Lauer microscopy. In this case, the reconstructed image, $\mathrm{O}_{\text {Lauer }}(\mathbf{r})$, is essentially the sum of the individual reconstructions that are characterised by equation 23 , and can be written such that,

$$
\mathrm{O}_{\text {Lauer }}(\mathbf{r})=\sum_{\mathrm{i}} \mathrm{E}^{\mathrm{m}}(\mathbf{r}) \mathrm{e}^{-\mathrm{j} \mathbf{k}_{\mathrm{i}} \cdot \mathbf{r}}
$$

Consequently, the process is a linear filtering operation, applied to the contrast function, that is characterised by the impulse response, $\mathrm{H}_{\text {Lauer }}(\mathbf{r})$,

$$
\mathrm{H}_{\text {Lauer }}(\mathbf{r})=2 \mathrm{k}_{0}^{2} \sum_{\mathrm{i}} \mathrm{G}_{\mathrm{NA}}(\mathbf{r}) \mathrm{e}^{\mathrm{j \mathbf {k } _ { \mathbf { i } } \cdot \mathbf { r }}}
$$

and transfer function, $\widetilde{\mathrm{H}}_{\text {Lauer }}(\mathbf{k})$, given by,

$$
\widetilde{\mathrm{H}}_{\text {Lauer }}(\mathbf{k})=2 \mathrm{k}_{0}^{2} \sum_{\mathrm{i}} \widetilde{\mathrm{G}}_{\mathrm{NA}}\left(\mathbf{k}-\mathbf{k}_{\mathrm{i}}\right)
$$

The transfer functions of a Lauer microscope are illustrated for the case of back-scatter and forwardscatter in figures 7a) and 7b) respectively. It is worth noting that the spectral measurements are not uniform in $\mathrm{k}$-space and in the algorithm published by Lauer an additional weighting is applied to "whiten" the spectral estimate [8]. Although this modifies the impulse response the axial and lateral bandwidths are unchanged and in our present analysis we will not consider this further. We note, however, that the bandwidths are exactly twice that obtained by a single holographic reconstruction and consequently, from equations 18 and 19 the axial and lateral extents of the impulse response (once again defined as the Nyquist distance) are halved. 


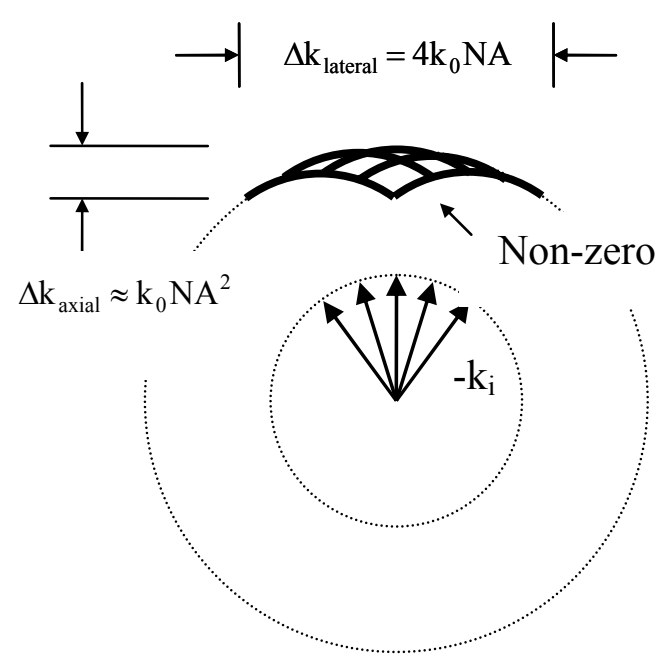

a)

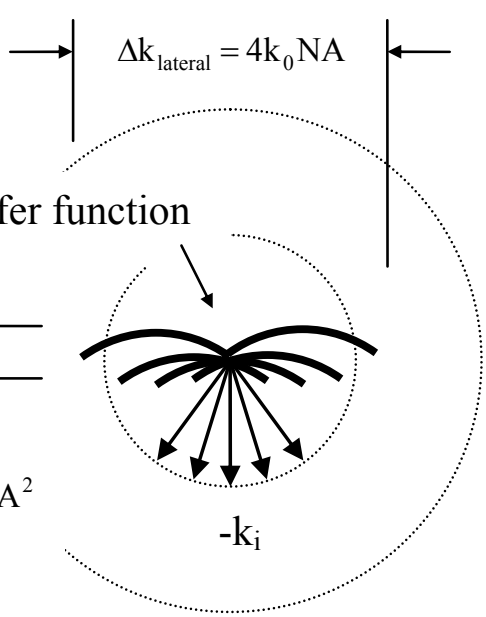

b)

Figure 7 Transfer function of Lauer Microscope in a) back-scatter and b) forward-scatter geometry

We also note that an ideal optical system, that is capable of sequentially illuminating the object with any spatial frequency and measuring any component of the scattered field that is on the surface of the Ewald sphere, has a transfer function, that can be written as the convolution,

$$
\begin{aligned}
\widetilde{\mathrm{H}}_{\text {ideal }}(\mathbf{k}) & =2 \mathrm{k}_{0}^{2} \int_{-\infty}^{+\infty} \delta\left(\left|\mathbf{k}_{\mathrm{i}}\right|-\mathrm{k}_{0}\right) \widetilde{\mathrm{G}}_{\text {ideal }}\left(\mathbf{k}-\mathbf{k}_{\mathrm{i}}\right) \mathrm{d}^{3} \mathrm{k}_{\mathrm{i}} \\
& =2 \mathrm{k}_{0}^{2} \widetilde{\mathrm{G}}_{\text {ideal }}(\mathbf{k}) \otimes \widetilde{\mathrm{G}}_{\text {ideal }}(\mathbf{k})
\end{aligned}
$$

From equation 13 the ideal transfer function is therefore,

$$
\mathrm{H}_{\text {ideal }}(\mathbf{r})=2 \mathrm{k}_{0}^{2} \operatorname{sinc}^{2}\left(\mathrm{k}_{0}|\mathbf{r}|\right)
$$

We note that since $\widetilde{\mathrm{G}}_{\text {ideal }}(\mathbf{k})$ is defined by the surface of the Ewald sphere the transfer function is bounded by a sphere of radius $2 \mathrm{k}_{0}$. This defines the cut-off frequency of an ideal monochromatic tomographic system for the case of weak scattering. In the same way we can consider axial systems of finite NA. If the NA of the illumination and receiver optics are equal we find the transfer functions of back-scatter and forward-scatter tomographic systems are given by, $\widetilde{\mathrm{H}}_{\mathrm{BS}}(\mathbf{k})$ and $\widetilde{\mathrm{H}}_{\mathrm{FS}}(\mathbf{k})$ respectively to be,

$$
\begin{gathered}
\widetilde{\mathrm{H}}_{\mathrm{BS}}(\mathbf{k})=2 \mathrm{k}_{0}^{2} \widetilde{\mathrm{G}}_{\mathrm{NA}}(\mathbf{k}) \otimes \widetilde{\mathrm{G}}_{\mathrm{NA}}(\mathbf{k}) \\
\widetilde{\mathrm{H}}_{\mathrm{FS}}(\mathbf{k})=2 \mathrm{k}_{0}^{2} \widetilde{\mathrm{G}}_{\mathrm{NA}}(\mathbf{k}) \otimes \widetilde{\mathrm{G}}_{\mathrm{NA}}(-\mathbf{k})
\end{gathered}
$$

With reference to figure 6 and equations 18 and 19 we note that for both forward and back-scatter tomographic systems, the axial and lateral extents of the impulse response are given by

$$
\begin{aligned}
\Delta \mathrm{r}_{\text {axial }} & =\frac{2 \pi}{\Delta \mathrm{k}_{\text {axial }}} \approx \frac{\lambda}{\mathrm{NA}^{2}} \\
\Delta \mathrm{r}_{\text {lateral }} & =\frac{2 \pi}{\Delta \mathrm{k}_{\text {lateral }}}=\frac{\lambda}{4 \mathrm{NA}}
\end{aligned}
$$

As we have previously remarked there are many ways to illuminate an object and subsequently measure the scattered field, however we will now show that equations 30-34 define the performance of all methods that realise monochromatic linear optical tomography.

\subsection{Confocal Microscopy}

An alternative strategy is to illuminate the object with a focussed monochromatic beam and measure the field that appears to originate from the focal point. A 3D image can be obtained in this way by scanning the focussed beam and receiver optics through object space. This is the confocal microscope, 
that was originally proposed by Minsky [7] and is shown schematically in figure 8. For the sake of brevity we will consider only the case of restricted NA in back-scatter.

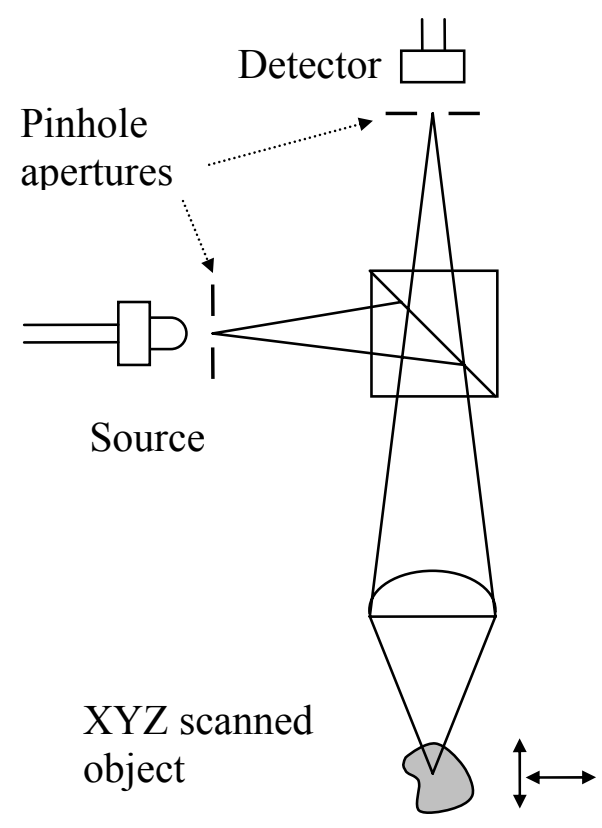

Figure 8 Concofal Microscope

Accordingly, the illuminating field at a point, $\mathbf{r}$, due to a beam that propagates through an optical system with restricted NA, that is focussed at point $\mathbf{r}^{\prime}$ will be,

$$
\mathrm{E}^{\mathrm{r}}(\mathbf{r})=\mathrm{G}_{\mathrm{NA}}\left(\mathbf{r}-\mathbf{r}^{\prime}\right)
$$

For the sake of comparison, we assume that we have a coherent detector (using a phase stepped reference for example [26]), from equation 21 the measured field is given by,

$$
E^{m}(r)=2 k_{0}^{2} \int_{-\infty}^{+\infty} G_{N A}^{2}\left(\mathbf{r}-\mathbf{r}^{\prime}\right) \Delta\left(\mathbf{r}^{\prime}\right) d^{3} r^{\prime}
$$

Hence following a similar process to before, a coherent, confocal reconstruction can be written as a linear shift invariant filtering operation,

$$
\mathrm{O}_{\mathrm{Con}}(\mathbf{r})=\mathrm{E}^{\mathrm{m}}(\mathbf{r})=\int_{-\infty}^{+\infty} \mathrm{H}_{\mathrm{Con}}\left(\mathbf{r}-\mathbf{r}^{\prime}\right) \Delta\left(\mathbf{r}^{\prime}\right) \mathrm{d}^{3} \mathrm{r}
$$

where the impulse response, $\mathrm{H}_{\mathrm{Con}}(\mathbf{r})$, is

$$
\mathrm{H}_{\mathrm{Con}}(\mathbf{r})=\mathrm{H}_{\mathrm{BS}}(\mathbf{r})=2 \mathrm{k}_{0}^{2} \mathrm{G}_{\mathrm{NA}}^{2}(\mathbf{r})
$$

Equation 38 shows that in the case of restricted NA in back-scatter, coherent, monochromatic confocal imaging and Lauer microscopy are equivalent. We note that, as discussed in section 4.2 additional weighting can be applied in k-space to "whiten" the spectral measurements obtained by a coherent, confocal microscope in the same manner as that originally proposed by Lauer [8]. A more complete analysis shows that the equivalence with Lauer microscopy remains for the cases of ideal coherent confocal systems and forward-scatter systems of restricted NA.

\subsection{Scanning Quasi-Monochromatic Interferometry}

In this section we consider another important illumination strategy that is usually referred to as Scanning While Light Interferometry (SWLI) and is often used for surface profilometry [5]. For the sake of our present comparison we will first consider such an instrument using quassi monochromatic illumination and we will refer to this as Scanning Quasi-Monochromatic Inteferometry (SQMI). Typically SWLI instrumentation uses a Mirau objective [27] as shown in figure 9a). 


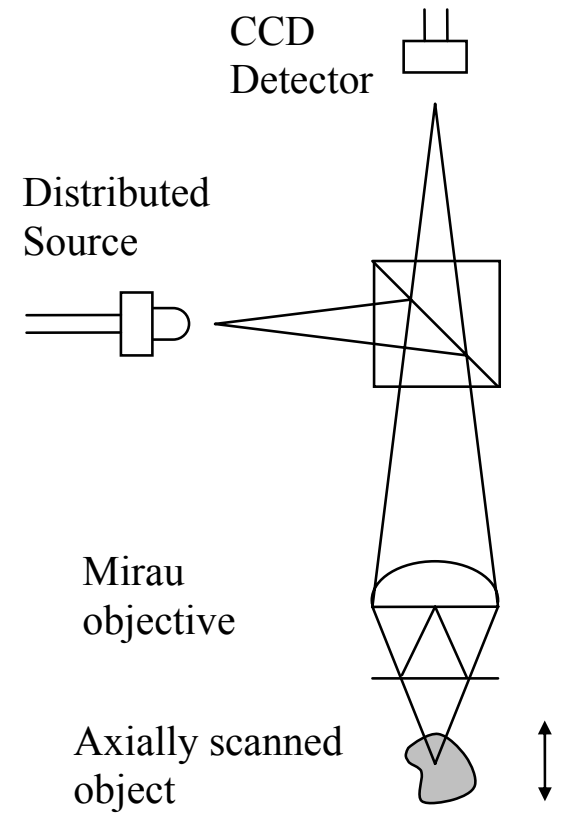

a)

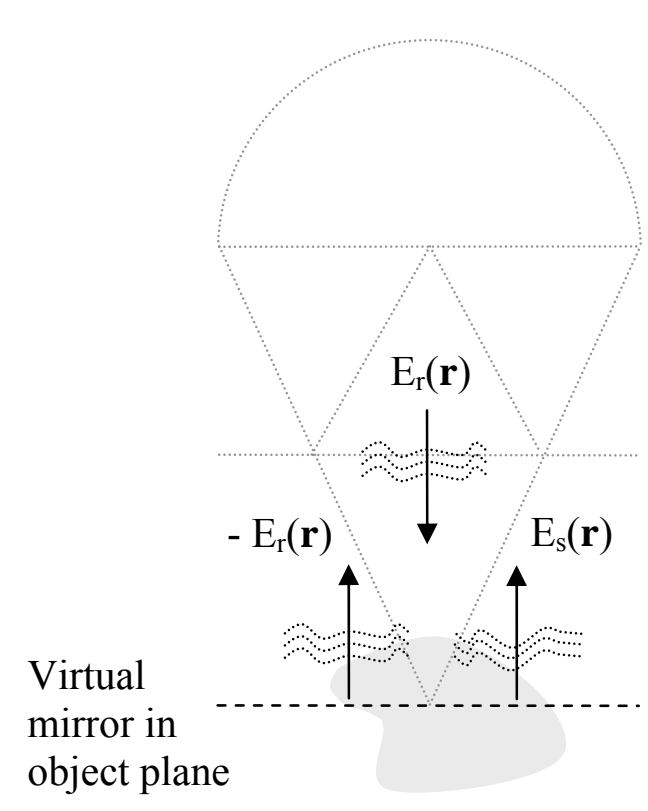

b)

Figure 9 SWLI a) Schematic b) Illumination and scattered fields.

With reference to Figure 9a) the object is illuminated by a distributed source and the scattered field in the object plane is imaged onto a CCD detector. The object is axially scanned through this plane. The Mirau objective however contains a semi-mirror that reflects a part of the illuminating wave and consequently the CCD records the interference of these two fields. SWLI is best understood by considering the interference that appears to originate from the object plane of the microscope. The geometry of the Mirau in effect places a virtual semi-mirror in the object plane of the imaging system as shown in figure $9 \mathrm{~b}$ ). In this plane the two beams consist of the field reflected from this mirror, that is assumed to be equal and of opposite sign to the illuminating field, $E^{r}(\mathbf{r})$, and the scattered field $E^{s}(\mathbf{r})$. It is noted however that the fields recorded by the instrument are filtered versions of these. Consequently, the recording is the imaged intensity of the interference between the measured scattered field, $E^{\mathrm{m}}(\mathbf{r})$, and the reflected illumination field $-\mathrm{E}^{\mathrm{r}}(\mathbf{r})$, such that

$$
\mathrm{I}(\mathbf{r})=\left|\mathrm{E}^{\mathrm{m}}(\mathbf{r})-\mathrm{E}^{\mathrm{r}}(\mathbf{r})\right|^{2}
$$

We will refer to this distribution as the interferogram. For the moment let us further restrict our discussion to monochromatic plane wave illumination, $\mathrm{E}^{\mathrm{r}}(\mathbf{r})=\mathrm{e}^{\mathbf{j} \mathbf{k}_{\mathrm{i}} \cdot \mathbf{r}}$, such that

$$
\mathrm{I}(\mathbf{r})=1+\left|\mathrm{E}^{\mathrm{m}}(\mathbf{r})\right|^{2}-\left[\mathrm{E}^{\mathrm{m}}(\mathbf{r}) * \mathrm{e}^{\mathrm{j} \mathbf{k}_{\mathrm{i}} \cdot \mathbf{r}}+\mathrm{E}^{\mathrm{m}}(\mathbf{r}) \mathrm{e}^{-\mathrm{j} \mathbf{k}_{\mathbf{i}} \cdot \mathbf{r}}\right]
$$

Clearly the terms in equation 38 are analogous to the usual terms in holography and for the case of restricted NA using a back-scatter geometry can be separated in the frequency domain by bandwidth. Consequently, we will neglect the first two terms in this expression and consider only the final term, $\mathrm{I}_{4}(\mathbf{r})$. From our expression describing the reconstructed field from an optical system of restricted NA (equation 21) we can obtain a reconstruction, $\mathrm{O}_{\mathrm{SQMI}}(\mathbf{r})$

$$
\mathrm{O}_{\mathrm{SQMI}}(\mathbf{r})=\mathrm{I}_{4}(\mathbf{r}) \mathrm{e}^{\mathrm{j} \mathbf{k}_{\mathbf{i}} \cdot \mathbf{r}}=2 \mathrm{k}_{0}^{2} \int_{-\infty}^{+\infty} \mathrm{G}_{\mathrm{NA}}\left(\mathbf{r}-\mathbf{r}^{\prime}\right) \mathrm{e}^{\mathrm{j} \mathbf{k}_{\mathrm{i}} \cdot\left(\mathbf{r}-\mathbf{r}^{\prime}\right)} \Delta\left(\mathbf{r}^{\prime}\right) \mathrm{d}^{3} \mathbf{r}^{\prime}
$$

It is noted that this expression is identical to equation 23 that describes the reconstruction from a single hologram with plane wave illumination and should not come as a surprise since our analysis closely resembles that of holography. It is straightforward to extend our analysis to the case of diffuse, quasi-monochromatic illumination. In this case, the interferogram is the sum of those obtained for 
each plane wave component in the illuminating field. Thus, for a back-scatter geometry of restricted $\mathrm{NA}$, following similar analysis we find, the transfer function of quasi-monochromatic SWLI is given by,

$$
\begin{aligned}
\widetilde{\mathrm{H}}_{\mathrm{SQMI}}(\mathbf{k}) & =2 \mathrm{k}_{0}^{2} \int_{-\infty}^{+\infty} \widetilde{\mathrm{G}}_{\mathrm{NA}}(\mathbf{k}) \widetilde{\mathrm{G}}_{\mathrm{NA}}\left(\mathbf{k}-\mathbf{k}_{\mathrm{i}}\right) \mathrm{d}^{3} \mathrm{k}_{\mathrm{i}} \\
& =2 \mathrm{k}_{0}^{2} \widetilde{\mathrm{G}}_{\mathrm{NA}}(\mathbf{k}) \otimes \widetilde{\mathrm{G}}_{\mathrm{NA}}(\mathbf{k}) \\
& =\widetilde{\mathrm{H}}_{\mathrm{BS}}(\mathbf{k})
\end{aligned}
$$

We note that this is exactly the transfer function obtained in the analysis of back-scatter confocal and Lauer microscopy geometries presented in sections 4.2 and 4.3. The transfer function and impulse response are illustrated in figure 10a) and 10b) respectively for the case of a monochromatic tomographic system operating at $550 \mathrm{~nm}$ with a $\mathrm{NA}=0.5$ in back-scatter.

a)
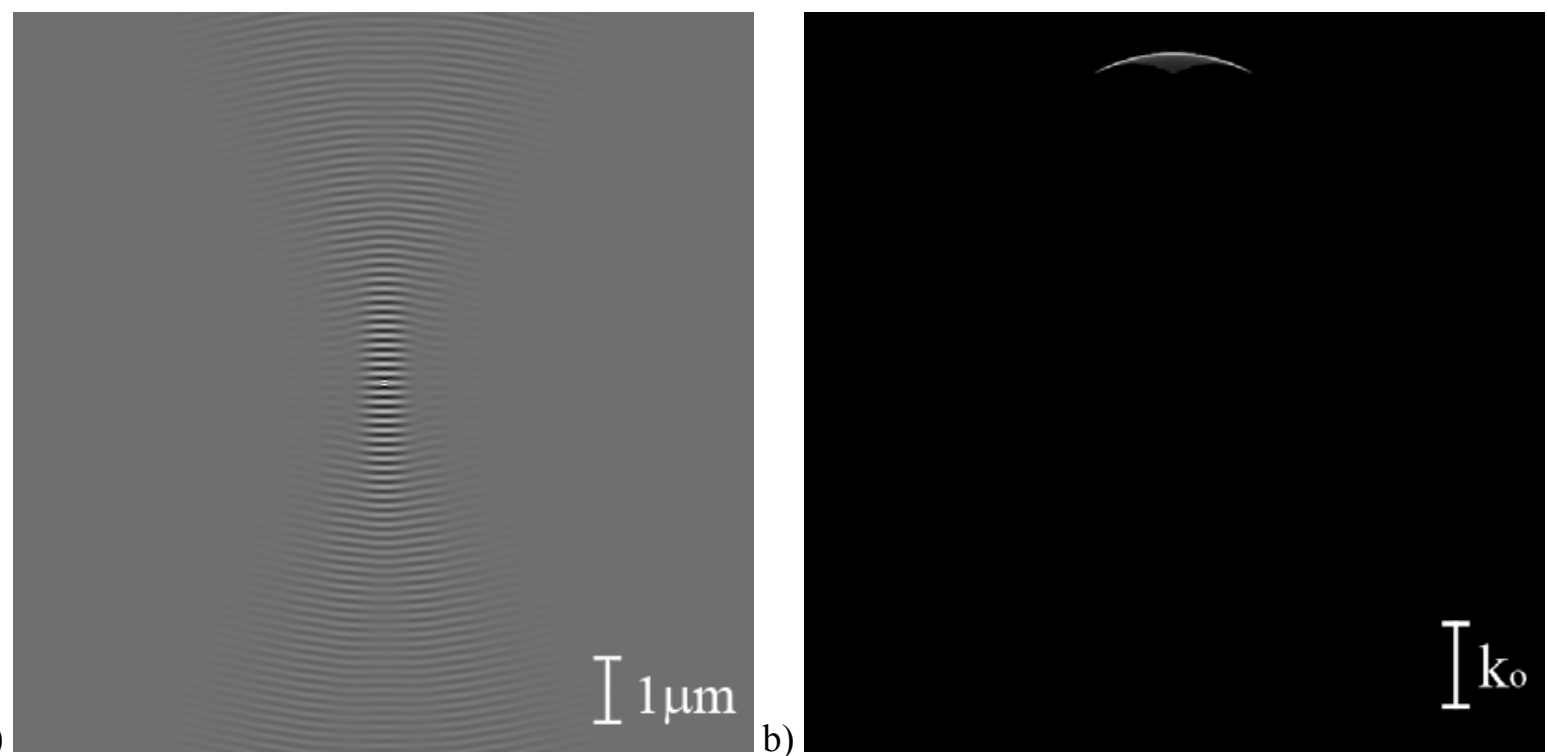

Figure 10. Characteristics of a monochromatic tomographic system a) Impulse response (real part) b) Transfer function (absolute value)

Comparing figure 10a) with figure 6a) it can be seen that the axial and lateral dimensions of the impulse response of a monochromatic tomographic recording is as predicted by equations 33 and 34, approximately half that of a reconstruction based the information provided by a single hologram. This is because the bandwidth is increased (doubled) as can be seen in figure 10b). It is also possible to increase the bandwidth of the reconstruction by using more than one wavelength and this is the basis of polychromatic optical tomography which is now discussed in more detail.

\section{Polychromatic optical tomography}

Under this heading we consider techniques that record the scattered field over a finite NA but exploit more than one wavelength. As expected there are many similarities with the monochromatic techniques described in the previous section, however, because the techniques have been developed independently a different terminology is generally used. Most of the techniques in this section (with the exception of polychromatic confocal microscopy) require coherent detection and can therefore be considered as derivatives of white light interferometry. White light path length scanning intereferometers were first used for tomographic purposes [16] and in this context the technique is called Optical Coherence Tomography (OCT). As object dimensions increase with respect to mean wavelength (and spatial resolution requirements decrease), there is a significant benefit in tuning the source or measuring the response to polychromatic illumination at a range of wavelengths simultaneously using a spectrometer. These techniques are collectively referred to as Spectral 
Optical Coherence Tomography (S-OCT). As we shall see, both techniques are closely related to Scanning White Light Interferometry (SWLI) and for this reason we will consider the response of this system first.

\subsection{Scanning White Light Interferometry (SWLI)}

In section 4.3 we considered the response of a path length scanning interferometer with monochromatic illumination. We noted that the transfer function of this system, $\widetilde{\mathrm{H}}_{\mathrm{SQMI}}(\mathbf{k})$, was exactly that of monochromatic confocal and Lauer microscopes operating in back-scatter, $\widetilde{\mathrm{H}}_{\mathrm{BS}}(\mathbf{k})$ and is related to the transfer function of a restricted aperture optical system, $\widetilde{\mathrm{G}}_{\mathrm{NA}}(\mathbf{k})$, by equation 42 . We also note that from equation 14 that $\widetilde{\mathrm{G}}_{\mathrm{NA}}(\mathbf{k})$ is a function of the wave number, $\mathrm{k}_{0}$, and since we are now concerned with more than one wavelength we will henceforth include this dependence explicitly, such that,

$$
\widetilde{\mathrm{G}}_{\mathrm{NA}}\left(\mathbf{k}, \mathrm{k}_{0}\right)=\delta\left(|\mathbf{k}|-\mathrm{k}_{0}\right) \operatorname{step}\left(\mathbf{k} . \hat{\mathbf{o}}-\mathrm{k}_{0} \sqrt{1-\mathrm{NA}^{2}}\right)
$$

Providing our detection system averages over an interval that is long compared to the coherence time of the source, the response of the system to polychromatic illumination system is simply the superposition of that of its spectral (temporal) components. Consequently, the polychromatic transfer function of a SWLI instrument is given by,

$$
\widetilde{\mathrm{H}}_{\mathrm{SWLI}}(\mathbf{k})=\int_{-\infty}^{+\infty} \mathrm{S}\left(\mathrm{k}_{0}\right)\left[2 \mathrm{k}_{0}^{2} \widetilde{\mathrm{G}}_{\mathrm{NA}}\left(\mathbf{k}, \mathrm{k}_{0}\right) \otimes \widetilde{\mathrm{G}}_{\mathrm{NA}}\left(\mathbf{k}, \mathrm{k}_{0}\right)\right] \mathrm{dk}_{0}
$$

where $\mathrm{S}\left(\mathrm{k}_{0}\right)$ is the spectral density of the source. The impulse response can therefore be written,

$$
\mathrm{H}_{\mathrm{SWLI}}(\mathbf{r})=2 \int_{-\infty}^{+\infty} \mathrm{k}_{0}^{2} \mathrm{~S}\left(\mathrm{k}_{0}\right) \mathrm{G}_{\mathrm{NA}}^{2}\left(\mathbf{r}, \mathrm{k}_{0}\right) \mathrm{dk}_{0}
$$

Once again a closed form solution to this equation is unknown to the Authors, however, the computed impulse response and transfer functions are illustrated for an instrument with a NA $=0.5$ and a source of uniform irradiance between $600-700 \mathrm{~nm}$ in figure 11a) and 11b).

a)
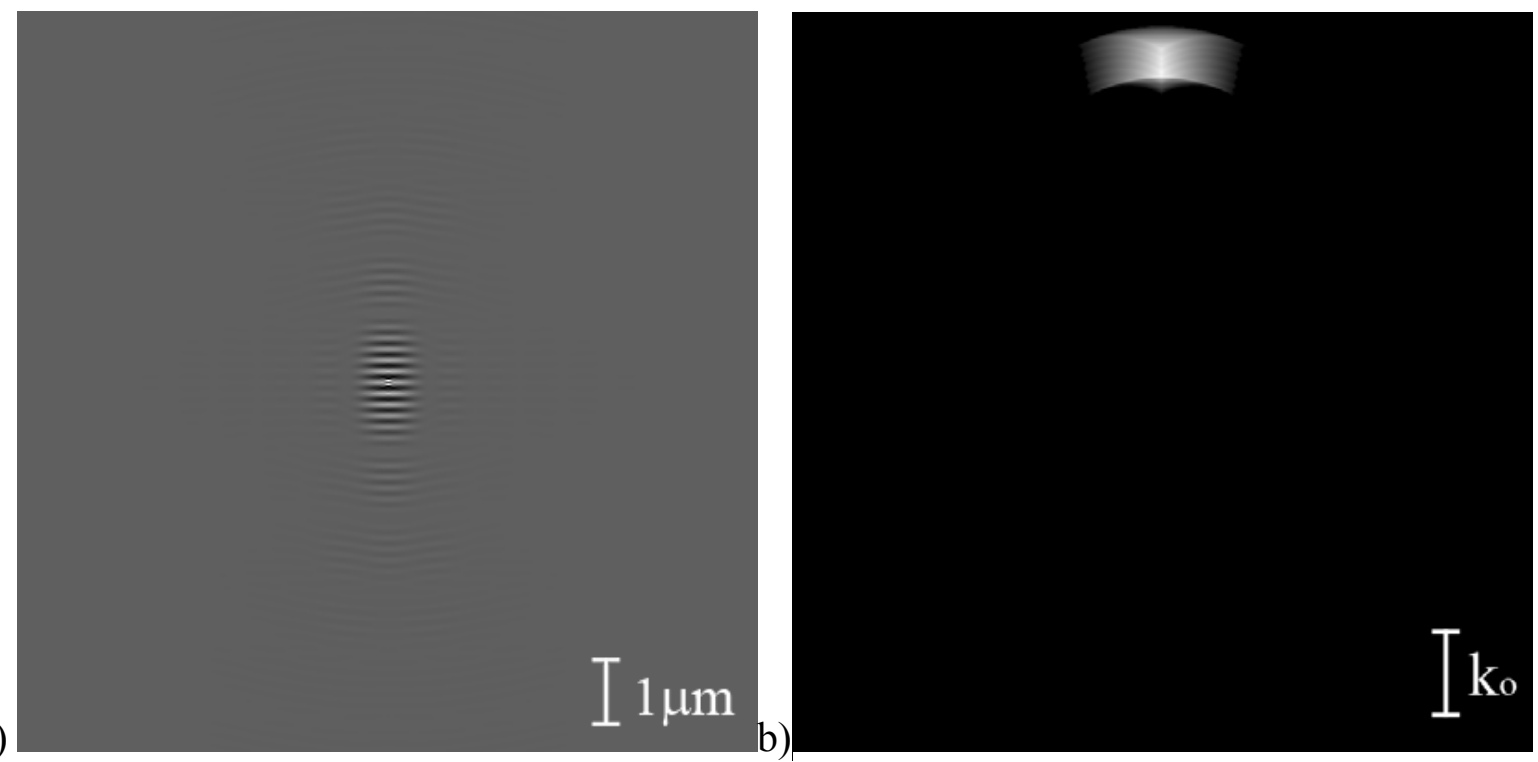

Figure 11. Typical SWLI characteristics a) Impulse response (real part) b) Transfer function (absolute value)

It can be seen that the temporal bandwidth of the system increases the spatial bandwidth of the reconstruction in the axial direction and decreases the axial extent of the impulse response. In a similar manner to before it is straightforward to show that the bandwidth of the reconstruction is given by,

$$
\Delta \mathrm{k}_{\text {axial }} \approx \overline{\mathrm{k}}_{0} \mathrm{NA}^{2}+2 \Delta \mathrm{k}_{0}
$$


where $\overline{\mathrm{k}}_{0}$ is the mean wave number and $\Delta \mathrm{k}_{0}$ is the range. The first term in equation 46 can be considered to be a contribution from focus while the second term results from increased temporal bandwidth. In some cases, for example in SWLI instruments, using large NA objectives and LED illumination the first term can be significant, however, the second term dominates when the full bandwidth of visible light is exploited. In this case the axial extent of the impulse response is given by,

$$
\begin{aligned}
& \Delta \mathrm{r}_{\text {axial }}=\frac{2 \pi}{\Delta \mathrm{k}_{\text {axial }}}=\frac{\pi}{\Delta \mathrm{k}_{0}} \\
& \approx \frac{\bar{\lambda}^{2}}{2 \Delta \lambda}
\end{aligned}
$$

where $\bar{\lambda}$ is the mean wave number and $\Delta \lambda$ is the range. The lateral resolution of the system is essentially identical to the monochromatic case. In practice, the benefits of polychromatic optical tomography becomes more apparent at low NA. This is generally referred to as Optical Coherence Tomography (OCT) and is discussed further in the following section.

\subsection{Optical Coherence Tomography (OCT)}

In contrast with most SWLI instruments OCT systems are generally used to investigate quite large objects for example the retina of the eye [10]. Although OCT can be considered as a SWLI instrument with a severely restricted aperture (and as such the transfer function is defined by equation 44), most practical systems have employed mechanical scanning to build up an image from single point data in a manner similar to that of a confocal microscope. In this case however, it is noted that the NA is usually small, the confocal effect is negligible and consequently no useful information can be retrieved by scanning in the axial direction. In OCT the extent of the impulse response is limited by the spectral bandwidth.

A typical system is shown in figure 12. In this case lenses are used to simultaneously focus the beams into the object and onto the reference mirror. Subsequently the beams are combined and reflected by a diffraction grating such that a focussed image at each wavelength is formed on the elements of a linescan CCD.

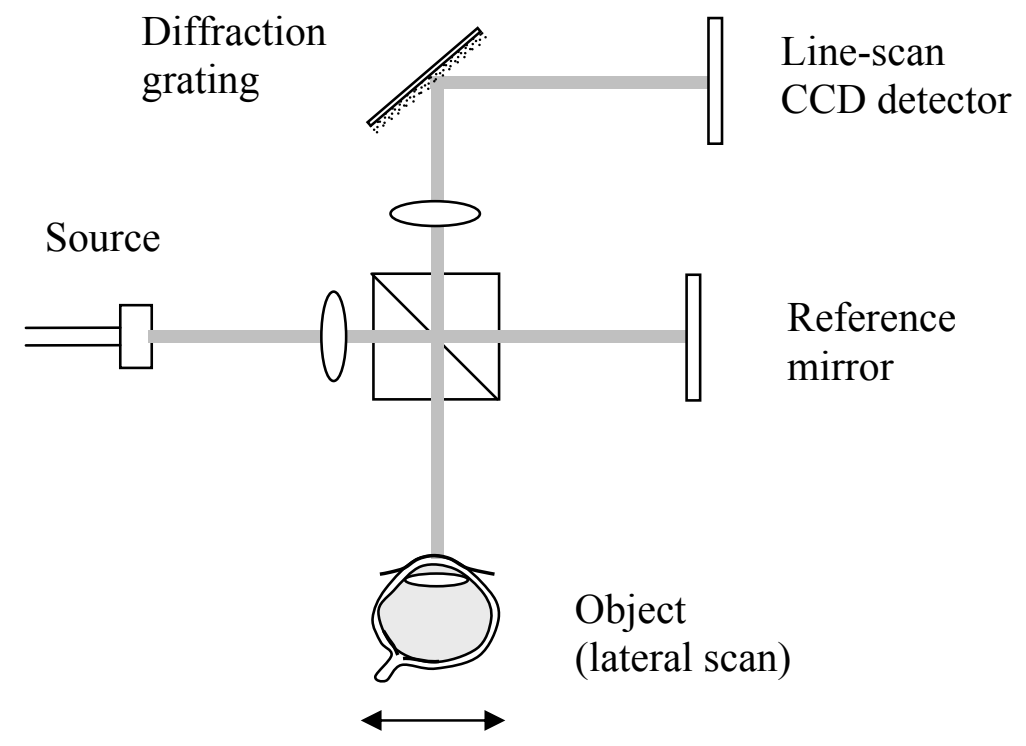

Figure 12 OCT Schematic

Because practical OCT systems use lateral mechanical scanning we base our analysis of OCT on that of the confocal microscope presented in section 4.2. For brevity we shall also limit our discussion to spectral OCT systems that essentially provide coherent detection at different wavelengths either by 
Wavelength Tuning Interferometry (WTI) or by dispersive means as in figure 12. It is noted once again that the impulse response of the optical system, the illuminating and scattered fields are all functions of wave number, $\mathrm{k}_{0}$. Accordingly, we will write this dependence explicitly such that for a system of restricted NA the illuminating field $\mathrm{E}^{\mathrm{r}}\left(\mathbf{r}, \mathrm{k}_{0}\right)$ at a point, $\mathbf{r}$, be due to a beam that is focussed at a point $\mathbf{r}^{\prime}$ is given by,

$$
\mathrm{E}^{\mathrm{r}}\left(\mathbf{r}, \mathrm{k}_{0}\right)=\mathrm{G}_{\mathrm{NA}}\left(\mathbf{r}-\mathbf{r}^{\prime}, \mathrm{k}_{0}\right)
$$

Let us assume that we measure the scattered field, which, from equation 21, can be written,

$$
\mathrm{E}^{\mathrm{m}}\left(\mathbf{r}, \mathrm{k}_{0}\right)=2 \mathrm{k}_{0}^{2} \int_{-\infty}^{+\infty} \mathrm{G}_{\mathrm{NA}}^{2}\left(\mathbf{r}-\mathbf{r}^{\prime}, \mathrm{k}_{0}\right) \Delta\left(\mathbf{r}^{\prime}\right) \mathrm{d}^{3} \mathrm{r}^{\prime}
$$

If we assume a spectral density $\mathrm{S}\left(\mathrm{k}_{0}\right)$ once again, a reconstruction using OCT can be written

$$
\mathrm{O}_{\mathrm{OCT}}(\mathbf{r})=\int_{-\infty}^{+\infty} \mathrm{S}\left(\mathrm{k}_{0}\right) \mathrm{E}^{\mathrm{m}}(\mathbf{r}) \mathrm{dk}_{0}=2 \int_{-\infty}^{+\infty} \int_{-\infty}^{+\infty} \mathrm{k}_{0}^{2} \mathrm{~S}\left(\mathrm{k}_{0}\right) \mathrm{G}_{\mathrm{NA}}^{2}\left(\mathbf{r}-\mathbf{r}^{\prime}, \mathrm{k}_{0}\right) \mathrm{dk}_{0} \Delta\left(\mathbf{r}^{\prime}\right) \mathrm{d}^{3} \mathrm{r}
$$

Consequently, impulse response $\mathrm{H}_{\mathrm{OCT}}(\mathbf{r})$, is

$$
\mathrm{H}_{\mathrm{OCT}}(\mathbf{r})=\mathrm{H}_{\mathrm{SWLI}}(\mathbf{r})=2 \int_{-\infty}^{+\infty} \mathrm{k}_{0}^{2} \mathrm{~S}\left(\mathrm{k}_{0}\right) \mathrm{G}_{\mathrm{NA}}^{2}\left(\mathbf{r}, \mathrm{k}_{0}\right) \mathrm{dk}_{0}
$$

Similarly the transfer functions of OCT and SWLI are also identically related

$$
\widetilde{\mathrm{H}}_{\mathrm{OCT}}(\mathbf{k})=\widetilde{\mathrm{H}}_{\mathrm{SWLI}}(\mathbf{k})=\int_{-\infty}^{+\infty} \mathrm{S}\left(\mathrm{k}_{0}\right)\left[2 \mathrm{k}_{0}^{2} \widetilde{\mathrm{G}}_{\mathrm{NA}}\left(\mathbf{k}, \mathrm{k}_{0}\right) \otimes \widetilde{\mathrm{G}}_{\mathrm{NA}}\left(\mathbf{k}, \mathrm{k}_{0}\right)\right] \mathrm{dk}_{0}
$$

We note however, that we have assumed 3D scanning in our analysis (as in confocal microscopy) and consequently the result is only strictly true in the plane of focus if $2 \mathrm{D}$ scanning is used. When the NA is restricted however, the impulse response is highly elongated in the axial direction (as described in Section 3) and equation 51 will represent the response of the system to a good approximation provided the object is within the depth of field.

Finally, we illustrate the transfer function and impulse response for a typical instrument with an $\mathrm{NA}=0.1$ and a source of uniform irradiance operating in the near infrared $(\bar{\lambda}=820 \mathrm{~nm} ; \Delta \lambda=20 \mathrm{~nm})$ in figures 13a) and 13b) respectively.
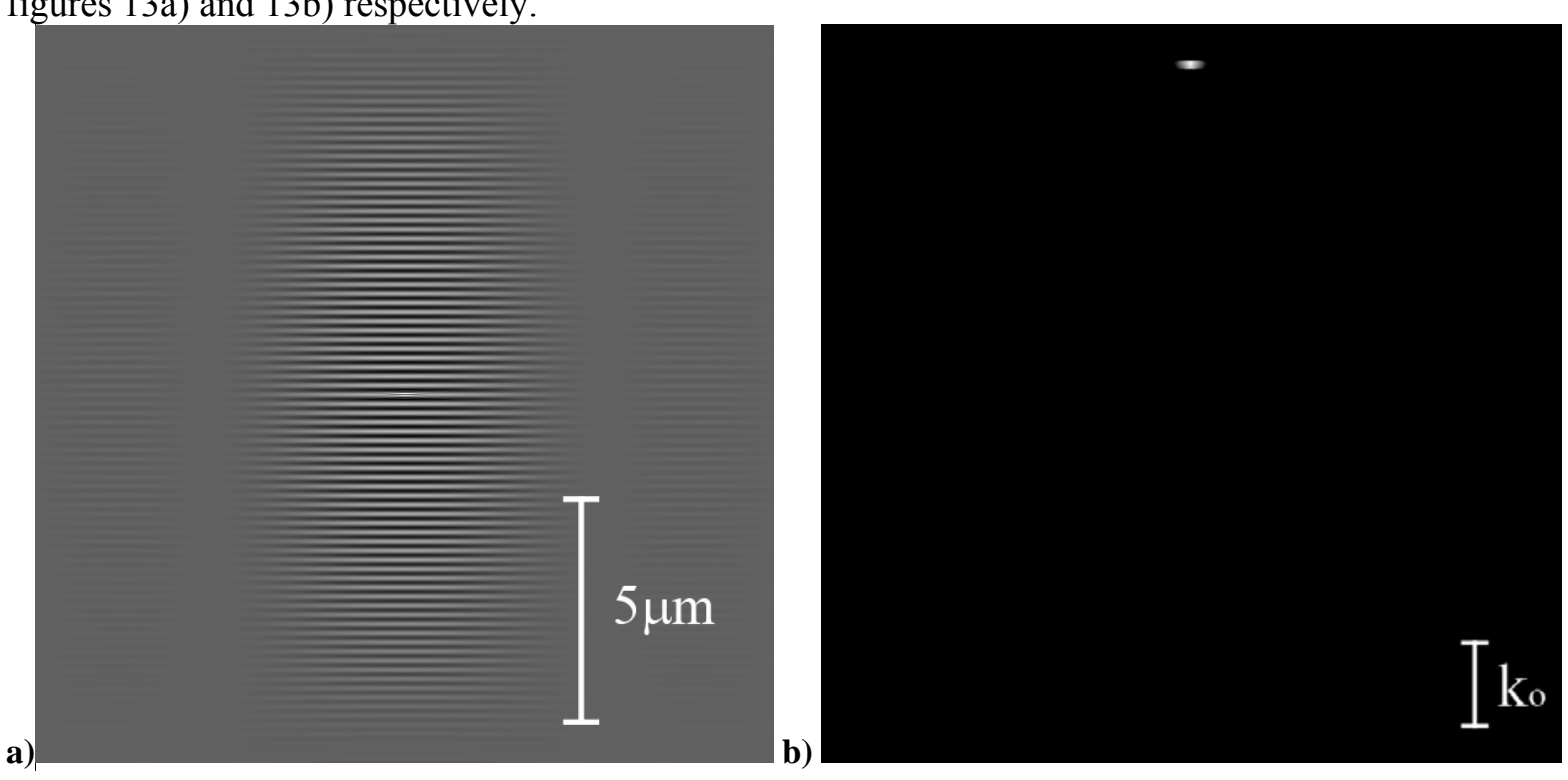

Figure 13 Typical OCT characteristics a) Impulse response (real part) b) Transfer function (absolute value)

In comparison with figure 10 we see that the impulse response is both wider and slightly longer than that of a monochromatic tomography system, however, the NA is considerably smaller. This allows OCT systems to provide high quality $3 \mathrm{D}$ images where monochromatic systems of large NA would be prohibited. 


\section{Conclusions and Discussion}

In this paper we have characterised 3D optical imaging techniques as 3D linear shift invariant filtering operations. Starting from first principles we begin by considering the scattered field to be the result of a linear filtering operation applied to a source distribution, that is characterised by the free-space Green's function. We then show that holographic reconstruction (or indeed any far-field optical system that produces a coherent image) can also be considered in the same terms. For an ideal imaging system (that collects the light scattered in all directions) we note that the 3D transfer function of this filter is an infinitely thin spherical shell, the Ewald Sphere, with a radius defined by the wave number and the 3D impulse response is a sinc function of the position. For the case of an axially symmetric system of restricted Numerical Aperture (NA) we show that the 3D impulse response rapidly elongates in the axial direction as the NA is reduced.

It is important to note that although the scattered field is a linear function of the source distribution, the latter is proportional to total field (i.e. both illuminating and scattered field). In the Born Approximation the contribution of the scattered field to the source term is neglected and we show that in this case imaging systems can be characterised as linear shift invariant filtering operations applied to the contrast of the refractive index (which effectively defines the object). Under the assumption that the Born approximation is valid, we characterise as filtering operations, holographic imagery, that reconstructs images of the contrast from recordings of the field scattered by a single illumination geometry, and distinguish this from monochromatic tomographic imagery, or Lauer microscopy, that combines the information in recordings made using more than one illumination geometry. Once again we note, the significance of NA, and the characteristics of monochromatic tomographic systems of restricted NA, that collect forward or back scattered light.

In our analysis we compare the response of Lauer microscopy (neglecting additional k-space weighting), with the 3D imagery offered by confocal microscopy (with coherent detection) and Scanning White Light Interferometry (SWLI), with quassi-monochromatic illumination, which we call Scanning Quassi-Monochromatic Interferometry (SQMI). We have shown that all these systems can be considered to provide tomographic imagery and have an identical response. The techniques differ most significantly in the way data is collected. Confocal microscopy uses a single point detector and requires full 3D scanning of the sample. Lauer microscopy makes use of modern area detectors and requires 2D (tilt) scanning of the illumination. It is easier to use than a confocal system in forward scatter since no critical alignment is required. SQMI uses an area detector and requires only 1D (axial) scanning of the object, however, it cannot operate in forward scatter. It can be concluded from our analysis that the choice of technique depends mainly on issues relating to ease of use.

We have also considered polychromatic optical tomography that synthesises an image from recordings of the scattered field made at more than one wavelength. We consider the response of SWLI instrumentation and that of Spectral Optical Coherence Tomography (S-OCT) both of which operate in backscatter. It is shown that the axial resolution of SWLI is defined both by NA and by the temporal spectrum of the source. At large NA the former dominates and the axial resolution is similar to its monochromatic counterpart, SQMI. At low NA, the converse is true and the axial resolution is inversely proportional to the spectral bandwidth. More over it is shown that the response of SWLI is equivalent to S-OCT at low NA. Once again the choice of technique is based on ease of use. It is clear however that for large samples (more than a millimetre or so) the high resolution axial scan required by SWLI is prohibitively time-consuming and the relatively low resolution lateral scanning used in SOCT is far more efficient.

We finish our analysis of 3D optical imaging returning briefly to a more general discussion of inversion of scattered field data to measure the optical properties of the object. As we mentioned in the introduction, the 3D optical imaging techniques studied here (and indeed many other optical techniques) simply measure the coherent response of an object to a set of known electromagnetic stimuli in the visible spectrum. The Lauer microscope and S-OCT techniques implement this approach directly but it is evident from our work that the same information can be retrieved using other 3D optical imaging techniques. We note that, under 
the Born Approximation images can be considered as linear shift invariant filtering operations that are applied to the refractive index contrast that defines the object. The Born Approximation is only valid for the case of weak scattering, however, and in practice its use is rarely justified. Nevertheless as we have seen, all the methods described in this paper provide images that can be considered to be synthesised assuming its validity. It seems that in many cases multiple scattering effects simply appear to be homogenous noise and can be neglected. In some cases, however, the noise is far from homogenous, and coherent artefacts can appear in the reconstruction. For example, in the case of SWLI instrumentation these effects can be seen in the vicinity of step discontinuities and in high aspect ratio surface profiles [28]. In these cases and other problems such as measuring droplet size in dense fuel sprays [19], nonlinear inversion strategies are required. This approach is considerably more computationally intensive than linear inversion and is beyond the scope of our present paper, however, further information can be found, for example in references [29].

\section{References}

[1] Trolinger J.D., 1975 Particle Field Holography, Opt. Eng 14,383-392.

[2] Coupland, J.M. and Halliwell, N.A. 1992, Particle image velocimetry: three-dimensional fluid velocity measurements using holographic recording and optical correlation. Applied Optics, 131(8), 1005-1007.

[3] Parker R J and Jones D G 1985 Holographic flow visualisation in rotating transonic flows Inst. Phys. Conf. Ser. No. 77: Session 3, 141-6.

[4] Vest C.M. 1979 Holographic Interferometry, John Wiley \& Sons, New York.

[5] Deck L. and De Groot P. 1994 High-speed noncontact profiler based on scanning white-light interferometry, Applied Optics 33(31), 7334-7338

[6] Mansfield D 2006 The distorted helix: thin film extraction from scanning white light interferometry, Proc. of SPIE - Vol. 6186, MEMS, MOEMS, and Micromachining II.

[7] Minsky M 1988 Memoir On Inventing The Confocal Scanning Microscope, Scanning 10(4) 128-138.

[8] Lauer V., 2002, New approach to optical diffraction tomography yielding a vector equation of diffraction tomography and a novel tomographic microscope, Journal of Microscopy 205(2) 165-176.

[9] Devaney A.J. 1982, A filtered backpropagation algorithm for diffraction tomography, Ultrasonic Imaging 4(4), 336-50.

[10] You JW, Chen TC, Mujat. M, Hyle Park B, and De Boer JF 2006 Pulsed illumination spectraldomain optical coherence tomography for human retinal imaging, Optics Express, 14(15), 6739-6748.

[11] De la Torre-Ibarra M H, Ruiz P D, and Huntley J M, 2006, Double-shot depth-resolved displacement field measurement using phase-contrast spectral optical coherence tomography, Optics Express 14(21), 9643-9656.

[12] Wilson T. 1989 Optical Sectioning in Confocal Fluorescent Microscopes, J. Microsc. 154, 143156.

[13] Wolf E., 1969, Three-dimensional structure determination of semi-transparent objects from holographic data, Optics Communications 1(4), 153-156.

[14] Dandliker R., and Weiss, K. 1970, Reconstruction of the three-dimensional refractive index from scattered waves, Optics Communications 1(7), 323-328.

[15] Fercher A. F., Bartelt H., Becker H., and Wiltschko E., 1979, Image formation by inversion of scattered field data: experiments and computational simulation, Appl. Opt. 18, 2427- 2439.

[16] Fercher AF, Drexler W, Hitzenberger CK, Lasser T, 2003, Optical coherence tomographyprinciples and applications, Reports on Progress in Physics, 66, 239-303.

[17] Gaskill J. D., 1978, Linear Systems, Fourier Transforms and Optics, John Wiley \& Sons.

[18] Goodman J W 1996 Introduction to Fourier Optics McGraw-Hill Companies Inc. New York.

[19] Lobera J. and Coupland J.M., 2007, Optical Diffraction Tomography in Digital Holographic 
Microscopy: Use of A-Priori Information. Proc. Int. Workshop on Digital Holographic Reconstruction and Optical Tomography for Engineering Applications, Loughborough (UK). ISBN 978094797456 5, pp.185-191.

[20] Streibl N., 1985, Three-dimensional imaging by a microscope, J. Opt. Soc. Am. A 2, 121- 127.

[21] Sheppard C. J. R. and Cogswell C. J., 1990, Three-dimensional image formation in confocal microscopy, J. Microsc. 159, 179-194.

[22] Born, M., Wolf E., 1999, Principles of Optics: Electromagnetic Theory of Propagation, Interference and Diffraction of Light. 7th edition, Cambridge University Press.

[23] Hariharan P. 1996, Optical holography: principles, techniques, and applications, 2nd edition, Cambridge University Press.

Cloud G., 1998, Optical Methods of Engineering Analysis, Cambridge University Press, Cambridge, UK.

[24] Abramowitz, M. and Stegun, I. A. (Eds.). 1972 Handbook of Mathematical Functions with Formulas, Graphs, and Mathematical Tables, 9th printing. New York.

[25] Coupland, J.M, Lobera J. and Halliwell N.A., 2000 Fundamental Limitations to the Spatial Resolution and Flow Volume that can be Mapped using Holographic Particle Image Velocimetry, Proc. of Europto/SPIE conference on Optical Diagnostics for Industrial Applications, Glasgow (UK), pp.90-102.

[26] Bruning J H, Herriott D R, Gallagher J E, Rosenfeld D P, White A D and Brangaccio D J 1974 Optical wavefront measuring interferometer for testing optical surfaces and lenses, Appl. Opt. 13, 2693-703.

[27] Delaunay G., 1953, Microscope interférentiel A. Mirau pour la mesure du fini des surfaces, Rev. Opt., Theor. Instrum. 32, 610-614.

[28] Gao F., Leach R.K., Petzing J. and Coupland J. M., 2008, Surface measurement errors using commercial scanning white light interferometers, Meas. Sci. Technol. 19 (2008)

[29] Belkebir K., Chaumet P.C., and Sentenac A., 2006 Influence of multiple scattering on threedimensional imaging with optical diffraction tomography, J. Opt. Soc. Am. A 23(3), 586-595

[30] Bulyshev AE, Souvorov AE, Semenov SY, Svenson RH, Nazarov AG, Sizov YE, Tatsis GP 2000, Three-dimensional microwave tomography. Theory and computer experiments in scalar approximation, Inverse Problems 16, 863-875.

[31] Devaney A J and Dennison M, 2003, Inverse scattering in inhomogeneous background media, Inverse Problems 19, 855-870. 